Article

\title{
Optimal Calibration Strategy of a Hybrid Electric Vehicle Equipped with an Ultra-Lean Pre-Chamber SI Engine for the Minimization of $\mathrm{CO}_{2}$ and Pollutant Emissions
}

\author{
Fabio Bozza, Vincenzo De Bellis $₫$, Enrica Malfi *®D, Luigi Teodosio $₫$ and Daniela Tufano \\ Dipartimento di Ingegneria Industriale, Università di Napoli Federico II, 80125 Napoli, Italy; \\ fabio.bozza@unina.it (F.B.); vincenzo.debellis@unina.it (V.D.B.); luigi.teodosio@unina.it (L.T.); \\ daniela.tufano@unina.it (D.T.) \\ * Correspondence: enrica.malfi@unina.it; Tel.: +39-081-7683264
}

Received: 15 June 2020; Accepted: 28 July 2020; Published: 3 August 2020

check for updates

\begin{abstract}
The complexity of modern hybrid powertrains poses new challenges for the optimal control concerning, on one hand, the thermal engine to maximize its efficiency, and, on the other hand, the vehicle to minimize the noxious emissions and $\mathrm{CO}_{2}$. In this context, the engine calibration has to be conducted by considering simultaneously the powertrain management, the vehicle characteristics, and the driving mission. In this work, a calibration methodology for a two-stage boosted ultra-lean pre-chamber spark ignition (SI) engine is proposed, aiming at minimizing its $\mathrm{CO}_{2}$ and pollutant emissions. The engine features a flexible variable valve timing (VVT) control of the valves and an E-compressor, coupled in series to a turbocharger, to guarantee an adequate boost level needed for ultra-lean operation. The engine is simulated in a refined 1D model. A simplified methodology, based on a network of proportional integral derivative (PID) controllers, is presented for the calibration over the whole operating domain. Two calibration variants are proposed and compared, characterized by different fuel and electric consumptions: the first one aims to exclusively maximize the brake thermal efficiency, and the second one additionally considers the electric energy absorbed by the E-compressor and drained from the battery. After a verification against the outcomes of an automatic optimizer, the calibration strategies are assessed based on pollutant and $\mathrm{CO}_{2}$ emissions along representative driving cycles by vehicle simulations. The results highlight slightly lower $\mathrm{CO}_{2}$ emissions with the calibration approach that minimizes the E-compressor consumption, thus revealing the importance of considering the engine calibration phase, the powertrain management, the vehicle characteristics, and its mission.
\end{abstract}

Keywords: virtual engine calibration; ultra-lean combustion; hybrid vehicle; 0D-1D engine modelling

\section{Introduction}

In the 2013, the Diesel-gate scandal erupted in the USA, flooding into Europe and worldwide, involving more than 11 million of vehicles produced by various car manufacturers [1]. The related rumors hit the entire automotive sector, leading to the idea that the traditional internal combustion engine (ICE), fed by fossil fuels, would disappear within a few years. A full-electric mobility, based on battery electric vehicles (BEVs), has been deeply promoted by politicians and public opinion, as the unique available solution to fulfill more and more stringent regulations [2].

At the same time, however, alternative propulsion architectures, such as hybrid electric vehicles (HEVs) and plug-in HEV (PHEVs), due to their lower costs and greater autonomy, continuously gained 
new market shares. Today, they represent much more than a temporary solution, filling the gap between standard ICE-powered vehicles and full electric mobility.

As known, the choice for the optimal electrification level of a vehicle requires to consider its entire life cycle, including the $\mathrm{CO}_{2}$ formation during the vehicle manufacturing and the one associated to electrical energy production and distribution. Under this point of view, the overall $\mathrm{CO}_{2}$ emissions from the two extreme antagonist vehicles (ICEs and BEVs) become comparable and are strongly dependent on the so called "energy-mix" utilized for electrical energy production [3,4]. In particular, BEV or PHEV have consistent benefits only when renewable energy sources are used, leading to the conclusion that an electric mobility cannot be considered as the only practical alternative due to the limited and country-dependent availability of renewable sources. Additionally, as already remarked, the higher ownership cost of the BEV, combined with the high battery charging time and the low autonomy, strongly limits their market acceptance and diffusion [5]. In the light of those concerns, rather than a pure-electric future, a scenario should be expected in the years to come characterized by variegated technologies that are best suited to the contest in which they are employed. This means that ICE-based vehicles, HEVs, PHEVs, BEVs and fuel cell-based vehicles will coexist in the market for a long time, pushing car manufactures to improve the propulsion system efficiency [6] with the aim to respect the stringent regulation in terms of $\mathrm{CO}_{2}$ and pollutant emissions.

Among the various pathways to improve the thermal efficiency of ICEs, various knock mitigation measures were attempted, such as the variable compression ratio [7], the cooled exhaust gas recirculation (EGR) [8], and the water injection [9]. Benefits of fuel consumption highly depend on the considered operating condition [10]. An increasing research work is nowadays devoted to ultra-lean combustion systems since they have the potential of simultaneously reducing the cylinder-out NOx emissions and the fuel consumption in the whole engine operating domain. Among the various concepts analyzed in the literature to sustain a lean combustion [11,12], such as fuel stratification [13,14], HCCI [15] and SACI [16], the employment of a pre-chamber (PC) ignition system is nowadays considered the most practical solution. In this architecture, a small PC volume is connected to the main chamber (MC) through some orifices. The combustion process starts in the PC and propagates in the MC one, in the form of hot burned jets ejected from the pre-chamber. The high turbulence intensity of these jets ignites the MC mixture and ensures a stable flame propagation even under extremely lean mixtures $[17,18]$.

The aim of this activity is to numerically analyze the calibration and the energy management strategy of a HEV equipped with an ultra-lean PC engine. The overall propulsion system under investigation is developed within the framework of the EAGLE H2020 project (https://h2020-eagle.eu/). The hybrid architecture and the components' sizing has been completely defined by the manufacturer and includes two electric motor/generator units, a battery, and two gearboxes. The system is designed to provide the maximum flexibility and a versatile series/parallel operation. A hybrid series-parallel architecture was chosen to combine the advantages of series powertrain in urban driving (including smooth takeoff) and of the parallel mode over rural/highway routes. Close-to-optimal operations for the ICE are guaranteed by a four-speed gearbox placed between the ICE and differential. A gearbox is preferred to a CVT power-split device to get a higher mechanical efficiency. The switch between series and parallel modes is handled by three clutches, which also help to decrease the mechanical losses when one of the motors is not used.

The thermal unit is equipped with an active pre-chamber ignition system and a flexible intake and exhaust VVT device. The very high air-flow demand of the ultra-lean architecture requires the adoption of a two-stage boosting system composed of a variable geometry turbocharger, coupled in series to an E-compressor (E-Comp). The presence of an electrically driven compressor implies that the energy input to the engine is not just the chemical energy given by the fuel. The energy input in the E-Comp also plays a role on the effective engine efficiency. The overall boost level can be then shared between the turbocharger compressor and the E-Comp, depending on the electrical energy input given to the second one. The latter is provided by the same battery pack of the hybrid propulsion system. In other words, the possibility to modify the overall boost level sharing between the LP compressor and 
the E-comp opens the way to two calibration variants. From one side, an intensive use of the E-Comp leads to a reduced backpressure and an improved brake thermal efficiency (BTE). A more frequent battery discharge/recharge is, however, to be expected along a driving mission. The opposite situation occurs when the boost level is mainly provided by the standard turbocharger. The main objective of this paper is hence to provide some insights on the calibration and energy management strategy, which provides the minimum $\mathrm{CO}_{2}$ and pollutant emission along representative vehicle missions.

To this aim, the pre-chamber engine under investigation is schematized in a 1D modeling framework. An "in-house" quasi-dimensional model is employed to reproduce the main in-cylinder phenomena, such as mixture formation, combustion, turbulence, and emissions. Its reliability has been validated in a prototype single-cylinder unit in previous activities $[19,20]$. Here, the same model, with few enhancements, is applied to foresee the behavior of the multi-cylinder engine as well.

The engine control parameters are set by coupling the 1D model to an external optimizer. The latter is instructed to maximize the engine BTE or, alternatively, a properly defined overall BTE, which also considers the electrical energy absorbed by the E-Comp (strategies \#1 and \#2). Optimization results are obtained at two engine speeds over a load sweep. They are then utilized to define a number of heuristic rules implemented within several PID controllers in the 1D model, mimicking the mathematical optimization procedure. This rule-based (RB) calibration strategy is preliminary validated through a direct comparison with the results of the external optimizer. Then it is utilized to compute two sets of complete engine performance maps, summarizing the calibration strategies following the maximum BTE and maximum overall BTE (OBTE), respectively. Finally, these two sets are embedded in a vehicle simulation able to quantify the $\mathrm{CO}_{2}$ and pollutant emissions over two different driving cycles, namely the worldwide harmonized light vehicles test cycle (WLTC) and a real driving emission (RDE) cycle. This framework is schematized and summarized in the flowchart in Figure 1.

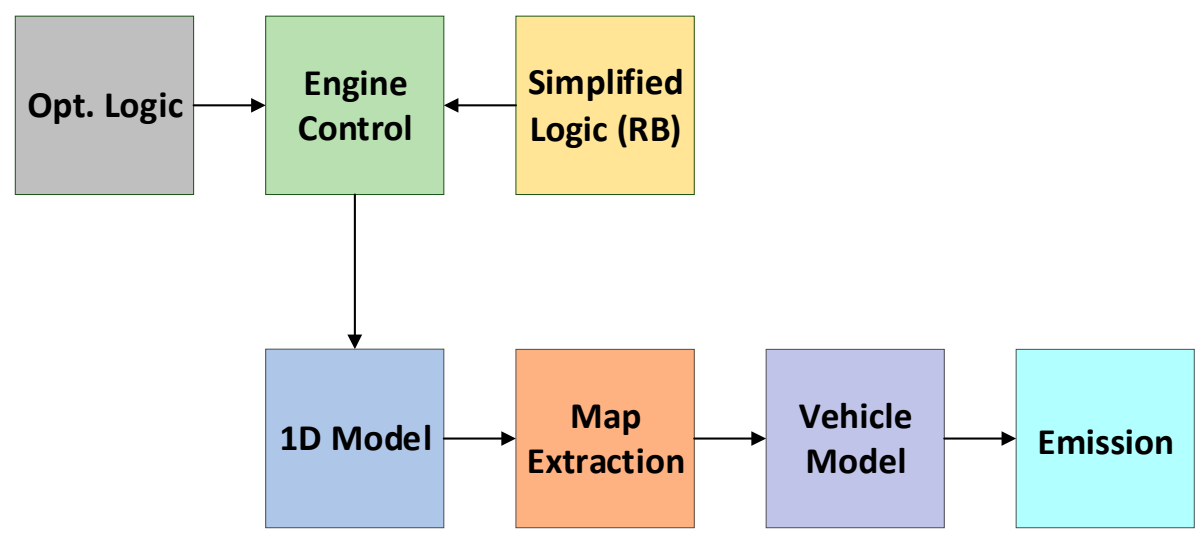

Figure 1. Flowchart of the methodology followed in the work.

The paper is organized as follows: firstly, the ultra-lean engine layout is described and the employed combustion model suitable for a pre-chamber engine is briefly recalled. Then, the numerical $\mathrm{RB}$ calibration procedure is illustrated and verified against the results of a multi-purpose optimizer. Finally, the energy management strategy developed by the authors is summarized [21] and the vehicle simulation outcomes are shown. In order to quantify the potential benefit of this HEV combined with this high-efficiency engine, the vehicle emissions are also compared against the current regulation limits (Euro 6d) and the $\mathrm{CO}_{2}$ European targets.

\section{Engine Description}

The SI engine under study, outlined in Figure 2, is a prototype power unit constituted by four cylinders and equipped with active pre-chambers. Main features of the investigated engine are summarized in Table 1. The PC features four orifices of $1 \mathrm{~mm}$ [22], and each cylinder has two intake and exhaust valves. The engine presents a cam phaser for both the intake and the exhaust camshaft. 
Moreover, two different cams profiles can be selected on the intake camshaft, allowing for an early valve closure (Standard Miller Lift-SML), or an extremely advanced valve closure (Extreme Miller Lift-EML) [22-24]. The latter is particularly suitable to limit the knock occurrence at high load.

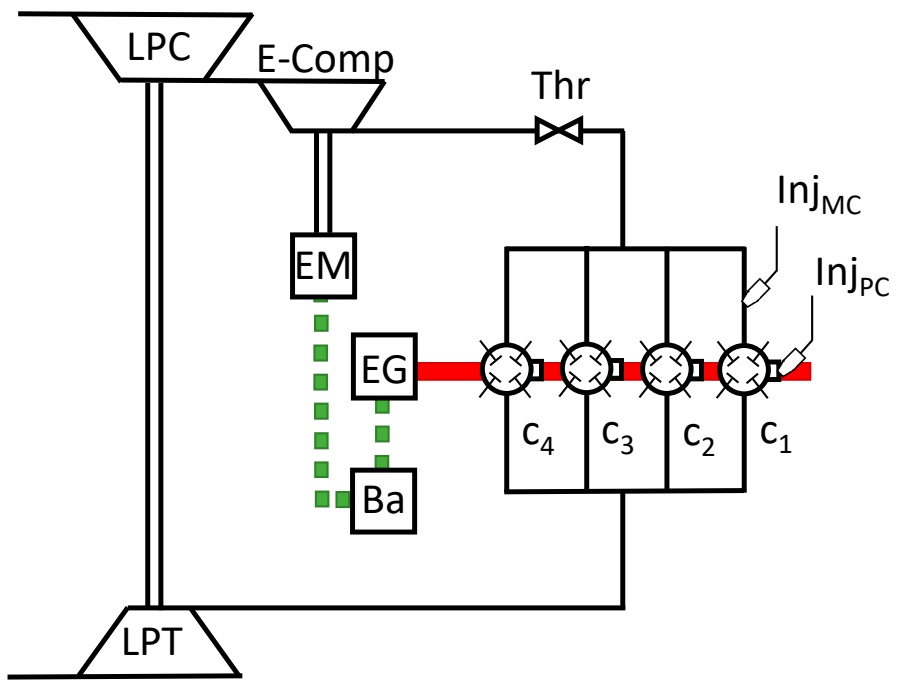

Figure 2. Schematic engine layout.

Table 1. Engine main features.

\begin{tabular}{cccc}
\hline \multicolumn{4}{c}{ Multi-Cylinder Pre-Chamber Engine } \\
\hline Bore & $76 \mathrm{~mm}$ & $\mathrm{~V}_{\text {pre-chamber }} / \mathrm{V}_{\text {TDC }}$ & $\sim 3 \%$ \\
\hline Stroke & $90 \mathrm{~mm}$ & PC hole number & 4 \\
Displacement & $1633 \mathrm{~cm}^{3}$ & PC hole diameter & $1 \mathrm{~mm}^{-1}$ \\
Compression ratio & 15.8 & $\mathrm{~A}_{\text {jet holes }} / \mathrm{V}_{\text {pre-chamber }}$ & $\sim 0.03 \mathrm{~cm}^{-1}$ \\
Fuel in main chamber & PFI, RON 95 & Intake Valve Opening & $344-370$ CAD (@0.7 mm lift) \\
Fuel in pre-chamber & DI RON 95 & Exhaust Valve Closure & $350-380$ CAD (@0.7 mm lift) \\
Pre-chamber volume & $\sim 1000 \mathrm{~mm}^{3}$ & Start of PFI & 140 CAD BTDC \\
Minimum PC injection duration & $300 \mu \mathrm{s}$ & Start of in-PC injection & 300 CAD BTDC \\
\hline
\end{tabular}

The engine is fed with liquid gasoline through four port fuel injectors located just upstream the intake valves (Inj $\mathrm{MC}_{\mathrm{C}}$ ), and four direct injectors located into each PC (InjPC). A two-stage boosting system provides the needed boosting level to match the prescribed load target. It is composed by a variable geometry turbocharger (LPC and LPT) and a high-pressure (HP) compressor (E-Comp), driven by an electric motor (EM). An electric generator (EG) mounted on the engine shaft provides the electrical energy to recharge the battery $(\mathrm{Ba})$ and to power the EM. The electric fluxes are shown as a dashed line in Figure 2.

As experimentally and numerically demonstrated [18-20], the introduction of a pre-chamber allows to strongly extend the lean burn limit of a conventional SI engine. This engine is designed to operate with very lean air/fuel mixtures to improve the engine efficiency and reduce the $\mathrm{NO}_{X}$ emission in a zone as wide as possible for the operating domain.

The presence of a high number of degrees of freedom makes the calibration of such a complex engine very challenging. In particular, the engine has nine control parameters, namely the relative air/fuel ratio $(\lambda)$ in the chambers (labelled as $\lambda_{P C}$ and $\lambda_{M C}$ ), the LPT rack position, the power input to the E-Comp, the intake and exhaust cam phases, the spark timing (or the combustion phasing, which can be represented by the angular position when half of the in-cylinder fresh charge is burned-50\% of mass fraction burned, $\mathrm{MFB}_{50}$ ), the throttle valve position, and the SML/EML cam profile. 


\section{D Engine Model Description and Validation}

Consistently with the engine geometry, a detailed 0D/1D scheme is developed in a commercial modeling framework. The description of the flow inside the intake and exhaust pipes is based on a 1D approach, whereas "in-house" 0D sub-models are used to reproduce the in-cylinder phenomena typical of this novel architecture, such as air/fuel mixture preparation, combustion, turbulence and heat transfer. Regarding PFI and DI injections, it is assumed that the 30\% of the injected fuel instantaneously evaporates, without considering spray evolution and liquid wall film formation. An empirical correlation is used for the estimation of the mechanical friction losses, dependent on engine speed, combustion phasing, and in-cylinder pressure peak. The steady-state flow coefficients for both the intake and the exhaust valves have been measured on a similar single-cylinder research engine and are here assigned to predict the flow permeability through the cylinder head $[19,20,22]$. A standard map-based approach is employed to reproduce the boosting system.

Each cylinder is schematized by two $0 \mathrm{D}$ volumes, a constant one (PC) and a variable one (MC), connected by means of an equivalent orifice. In particular, the overall cross-sectional area of the four holes is assigned in the above orifice. The orifice model also takes into account a discharge coefficient, fixed to a constant value of 0.65 , which gives the best agreement with the PC-MC pressure difference along the compression stroke. According to a classical filling-emptying technique, mass and energy equations are solved in both volumes to estimate the mass exchange between them, based on pressure difference and overall effective cross-sectional area. The combustion process is described by a rearranged quasi-dimensional fractal model, developed by the authors in the last years [25]. It is based on a two-zone (burned and unburned gases) approach, and it is applied in a similar way in both MC and PC. In this context, the overall burn rate can be expressed as the sum of "classical fractal flame propagation" and a "turbulent jet combustion," expressed by the Equation (1).

$$
\left(\frac{d m_{b}}{d t}\right)_{\text {overall }}=\left(\frac{d m_{b}}{d t}\right)_{\text {fractal }}+\left(\frac{d m_{b}}{d t}\right)_{\text {jet }}
$$

The first term of Equation (1), applied in both PC and MC, describes the burning rate through the fractal approach under the hypothesis that the flame front locally propagates at a laminar speed, $S_{L}$, and that the combustion process is promoted by the turbulence-induced flame wrinkling, $\Sigma$, leading to the following expression:

$$
\left(\frac{d m_{b}}{d t}\right)_{\text {fractal }}=\rho_{u} A_{L} S_{L} \Sigma
$$

where $\rho_{u}$ is the unburned gas density and $A_{L}$ is the laminar flame front area. The second term of Equation (1) models the burning rate contribution in the MC due to the turbulent jets, under the hypothesis that the jets entrain fresh charge (air and fuel) and rapidly burn it, in turn releasing heat. This is described by the equations below:

$$
\begin{gathered}
\left(\frac{d m_{b}}{d t}\right)_{j e t}=\frac{d m_{b, e n t r}}{d t}=\frac{m_{\text {entr }}-m_{b, e n t r}}{\tau} ; \quad \tau=\frac{\Lambda_{T}}{S_{L}} \\
\frac{d m_{\text {entr }}}{d t}=c_{j e t} \dot{m}_{j e t} \sqrt{\frac{\rho_{\mathrm{PC}}}{\rho_{\mathrm{MC}}}}
\end{gathered}
$$

The jet-induced burning rate is assumed proportional to the difference between the current entrained mass $\left(m_{\text {entr }}\right)$ and its burned portion $\left(m_{b, e n t r}\right)$. The Equation (3) resembles the well-known eddy burn-up approach [26,27], for which $\tau$ is a characteristic time scale. This last is calculated as the ratio between the Taylor length scale, $\Lambda_{T}$, and the laminar flame speed, $S_{L}$. Finally, the total entrained mass, $m_{\text {entr }}$ is computed by the integration of its time derivative, according to Equation (4), which in turn is estimated by the semiempirical correlation reported in [28]. This entrained mass is a function of 
the mass flow rate from the PC, $\dot{m}_{j e t}$, on a tuning constant $c_{j e t}$ ad on the density ratio between PC and MC. Equally, the burned entrained mass, $\left(m_{b, e n t r}\right)$, is estimated by the integration of Equation (3).

The summarized combustion model requires the evaluation of a few parameters-namely the flame wrinkling $\Sigma$ and the Taylor scale $\Lambda_{T}$-which depend on the turbulent field established in the combustion chamber. An "in-house" developed phenomenological K-k-T turbulence sub-model is employed to estimate those quantities [29]. The turbulence model takes into account the energy flux transferred from the mean flow kinetic energy, $\mathrm{K}$, to the turbulent one, $\mathrm{k}$. The model includes proper balance equations for tumble and swirl angular momentum, T and S. The model is applied to both $\mathrm{PC}$ and $\mathrm{MC}$, and also considers the turbulence production induced by the incoming/outcoming flow through the orifices [20].

The description of the knock phenomenon is based on the calculation of the auto-ignition (AI) delay time of the air/fuel mixture in the unburned zone. In particular, AI is carried out with a tabulated approach [30], employing preliminary auto-ignition chemical kinetic simulations performed in a homogeneous reactor at a constant pressure. The kinetic scheme adopted for the estimation of the table includes five elements, 201 species, and 1548 reactions [31] and an additional skeletal sub-mechanism for toluene oxidation to handle a toluene reference fuel. The AI time, $\tau_{A I}$, is stored in the table as a function of pressure, temperature, equivalence ratio, and residual content. As known, due to the presence of the cyclic dispersion, faster-than-average pressure cycles may induce the presence of a stochastic soft-knock, even when the average cycle is working in a safe, non-knocking combustion. Since, in the adopted approach, the AI integral, Equation (5), is computed on the average cycle, a small safety margin is applied, and the knock event is considered to occur when the AI integral exceeds a tunable lower-than-unity threshold level.

$$
\int \frac{d t}{\tau_{A I}}
$$

The model allows to estimate the regulated cylinder-out emissions, namely $\mathrm{CO}, \mathrm{HC}$, and NO. For the evaluation of $\mathrm{CO}$ and $\mathrm{NO}$, a multizone-zone approach is applied in both $\mathrm{PC}$ and $\mathrm{MC}$ to estimate the burned gas stratification. Each burned parcel is compressed/expanded adiabatically according to the in-cylinder pressure. The estimated local zone temperatures are employed within the well-known extended Zeldovich mechanism for the estimation of the NO [32], whereas the CO is computed with a two-step reaction scheme [33]. The experimental findings show that in ultra-lean engines the noxious emissions are mainly composed of $\mathrm{NO}_{2}$, therefore in the adopted approach the $\mathrm{NO}$ production derived by the Zeldovich mechanism is assumed to completely oxidize the $\mathrm{NO}_{2}$ when the burned gases evolve along the exhaust pipes. Concerning the $\mathrm{HC}$, the crevice contribution and the post-oxidation is considered only for the MC, neglecting the PC contribution. In the employed model, the HC filling and emptying within the crevice regions is followed [34]. The temperature in this volume is considered to be the same as the piston wall, while the pressure equals the one in the cylinder. When unburnt fuel is released from the crevices, a proper boundary layer temperature is computed, at which the released HC partly oxidize according to the kinetic rate proposed in [35].

The quasi-dimensional combustion model was tuned with reference to a research engine $[19,20]$, having geometrical and architectural characteristics similar to the four-cylinder engine under study for which experimental data are not yet available. The model was tuned by a trial-and-error procedure aiming to obtain the best agreement with pressure signals in both PC and MC. A unique set of tuning constants was selected for all the operating conditions, characterized by very different speeds, loads, and air/fuel ratios ( $\lambda$ up to 2.4 in the main chamber) $[19,20]$.

\section{Optimization Approach for Engine Calibration}

With the aim of calibrating the engine, a value for each of the nine control parameters listed in the "Engine Description" section must be specified in each operating condition. The purpose of this task is the optimization of the engine performance over the entire operating domain. 
The calibration procedure here targets the maximum possible efficiency, fulfilling several constraints-i.e., maximum pressure inside MC and PC, knock intensity, turbo speed, boost level, E-Comp power, internal EGR amount, etc.-prescribed to confine thermal and mechanical stresses and operating reliability for the engine and for its components.

To find the optimal set of the control parameters at variable speeds and loads, an automatic optimization is performed, realized by using an external multi-purpose optimizer. The optimization is performed considering two different control strategies with differentiated fuel and electric consumptions. Particularly, the first control strategy (strategy \#1) aims to maximize the conventional BTE, namely:

$$
\mathrm{BTE}=\frac{P_{\mathrm{ICE}}}{\dot{m}_{f} \mathrm{LHV}}
$$

where $P_{I C E}$ is the brake power at the engine shaft, $\dot{m}_{f}$ is the total injected fuel flow rate, and LHV is the lower heating value of the fuel.

The second one (strategy \#2) is oriented to the optimization of the overall BTE:

$$
\mathrm{OBTE}=\frac{P_{\mathrm{ICE}}-P_{\mathrm{HPC}} / \eta_{\mathrm{HPC}}}{\dot{m}_{f} \mathrm{LHV}}
$$

where $P_{\mathrm{HPC}}$ is the mechanical power at the E-Comp shaft and $\eta_{\mathrm{HPC}}$ is the electro-mechanical efficiency of the E-Comp. Based on its definition, the OBTE also considers the electric energy required by the E-Comp and drained from the battery.

For both examined control strategies, the optimization is realized along a brake mean effective pressure (BMEP) sweep at two different engine speeds, namely 2000 and $3000 \mathrm{rpm}$. Low/medium engine rotational speeds are selected because of their relevance regarding the vehicle homologation cycles. The load sweep is automatically realized by alternatively maximizing or minimizing the BMEP, together with the BTE/OBTE, hence performing four different multi-objective problems for each engine speed. Each optimization problem hence identifies a single branch of the BTE/BMEP or OBTE/BMEP Pareto frontiers.

The selected optimization tool is the genetic MOGA-II algorithm, which represents the best choice for the case of a multi-variable/multi-objective problem. The nine control parameters previously listed, apart from the relative air-fuel ratio in the main chamber which is always metered to fix a value of two, represent the independent variables of the optimization problem. Each variable is changed within a prefixed allowable range. The selection of the intake valve lift profile is indeed handled by introducing a fictitious discrete variable, associating values of 0/1 to the Standard/Extreme Miller valve lifts, respectively.

The logical scheme of the optimization process was already reported in a previous author's work [36], and here it is partially modified by the addition of SML/EML and EVC (exhaust valve closure) variables. The optimizer algorithm, at each step with an iterative process, selects the eight engine control variables, passing them to the $1 \mathrm{D}$ engine model to evaluate the variables of interest. At the end of each simulation, the estimated values of the objective function are returned to the optimizer to start the next iteration. This process pursues as long as the optimal levels, being part of the Pareto frontier, are achieved.

As said, the optimizer evaluations are post-processed and filtered to check that some monitored variables do not exceed prescribed threshold levels. As an example, the maximum in-cylinder pressure is limited to $180 \mathrm{bar}$, the maximum plenum pressure to $4.5 \mathrm{bar}$, the maximum E-Comp power to $10 \mathrm{~kW}$, the maximum $\mathrm{AI}$ integral to 0.8 , etc.

\section{Assessment between RB and Optimizer Calibrations}

The previously described optimization procedure is too time consuming to be directly employed to compute the complete engine performance map. To speed up the numerical calibration, a set 
of heuristic rules were defined to reproduce as best as possible the behavior of the mathematical optimization (rule-based calibration). The RB was applied by including a network of logical switches, PID controllers, and "math functions" in the 0D/1D environment.

Preliminarily, a prefixed running line was chosen on the LPC map as a tradeoff between an appropriate surge margin and a suitably high-pressure ratio to avoid an excessive E-Comp shaft power absorption (below $10 \mathrm{~kW}$ ). Under these operating conditions, the E-Comp running line was directly identified to reach the HP boost level necessary to fulfill a prescribed full load (FL) target, depicted in red in Figure 3. By changing the LPC running line, a different sharing of the overall boost level between the LPC and the E-Comp would have been accordingly identified. However, whatever the choice for the LPC running line, at very high engine speeds (usually over $4500 \mathrm{rpm}$ ) for the selected boosting devices, the constraints on the maximum E-Comp power and/or maximum boost level could not be fulfilled. For this reason, the only way to achieve the FL target was to inject more fuel in the main chamber, resulting in a $\lambda_{\mathrm{MC}}<2$. The L2 line in Figure 3 identifies the BMEP region below which a $\lambda_{\mathrm{MC}}=2$ operation can be sustained (light yellow domain), while the area above the L2 line defines the operating domain with $\lambda_{\mathrm{MC}}<2$ (highlighted in orange).

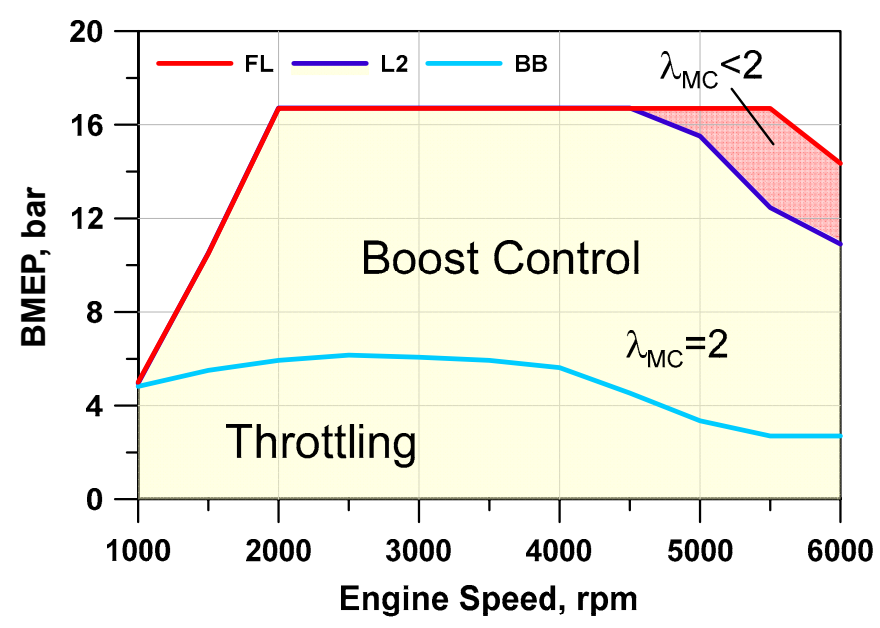

Figure 3. BMEP-rpm map containing the BMEP target (FL), and the L2 and BB lines.

In the low-load region, an additional line is defined corresponding to the BMEP levels reached with the LPT fully open and no power given to the E-Comp, named Base Boost Line (BB in cyan in Figure 3). In these conditions, the E-Comp is bypassed, acting on dedicated control valves. Above the $\mathrm{BB}$ line, the engine load is regulated by a progressive closing of the LPT and a proportional increase of the E-Comp power. Below the BB line, the LP and HP compressor settings continue to be fixed, while the load is only regulated by the progressive closure of the throttle valve.

Concerning the VVT position of intake and exhaust camshafts, simple rules are defined to select an advanced IVC (intake valve closure) at low load to have a pumping losses reduction. The intake valve advancing is, however, limited at very low load to avoid an internal EGR rate above $20 \%$. In this way, a stable combustion process can be guaranteed in the real engine at the expense of a certain pumping losses increase.

In the mid-load range, the intake VVT is moved towards a later IVC to allow for increased air flow rates, while, at a very high load, an early IVC is once again preferred to reduce the effective compression ratio for knock control. The exhaust valve is accordingly controlled to limit the valve overlap and/or to increase the expansion work at a low load.

The actual positions of the BB and L2 lines also depend on the selection of the intake lift profile, namely the SML or the EML. To reduce pumping losses, the EML is always specified when the computed airflow rate is below $100 \mathrm{~kg} / \mathrm{h}$. By analyzing the results of the previous mathematical optimization, it was indeed found that for strategy \#1 (max BTE), the SML is only selected below a 
specific BMEP limit (namely 6.5 bar), while it is always selected above $100 \mathrm{~kg} / \mathrm{h}$ airflow rate in the strategy \#2 (max OBTE). This can be easily explained by observing that the SML requires a lower boost level for the same load. For this reason, a lower E-Comp power is required, which improves the OBTE. Moreover, to better reproduce the optimization results, a different running line is selected for the strategy \#2, moving the boost sharing mainly on the LPC and reducing the E-Comp contribution.

The proposed rule-based methodology is quite refined to provide engine operations very close to the optimal BTE and OBTE, as confirmed in the following. In addition, the advantage of this RB strategy is related not only to its easy implementation in the used 1D software, but also to its much faster execution compared to a set of multi-objective optimizations.

To quantify the reliability of the two implemented calibration strategies, their outcomes were compared to the optimization results. The optimization outcomes are shown in the next figures with open symbols and label Opt and refer to the outcomes belonging to the Pareto frontier.

Figure 4 reports the comparisons of BTE and OBTE at $2000 \mathrm{rpm}$ for the considered calibration strategies (\#1 and \#2). In both cases, a considerable agreement was realized along the BMEP sweep between Opt and RB procedures. Figure 4 also shows the potentiality of the analyzed engine architecture to reach a very high BTE level of about $50 \%$ at the higher load. In the latter conditions, lower OBTE is reached due to the relevant power request by E-Comp (see OBTE definition in Equation (5)). Indeed, OBTE assumes a maximum value around $42 \%$ at medium loads, with a slight reduction at the highest BMEP levels.

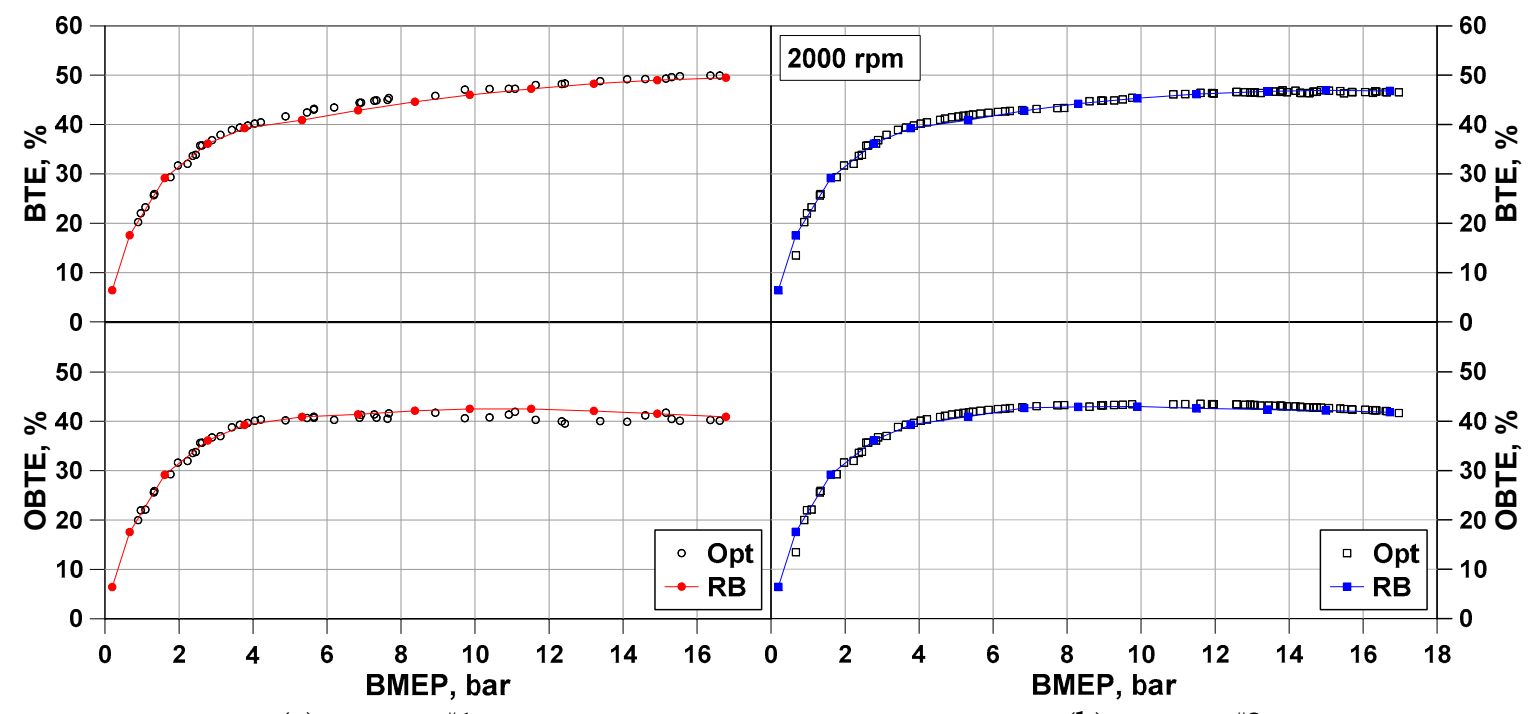

(a) strategy \#1

(b) strategy \#2

Figure 4. Brake thermal efficiency (BTE) and overall brake thermal efficiency (OBTE) comparison in a BMEP sweep at 2000 rpm for strategies \#1 (a) and \#2 (b).

Quite satisfactorily, RB/Opt agreements were found for the most relevant engine calibration variables depicted in Figures 5 and 6. The first assessment regards the intake valve strategy and the intake/exhaust valve timings, which are plotted in Figure 5. Considering the strategy \#1, the Extreme Miller was mainly preferred all along the BMEP sweep, while in the strategy \#2 at medium/high loads, the Standard Miller strategy was chosen.

On a general viewpoint at both low and high loads, IVC is selected near to the most advanced timing setting. This option, as said, is preferred for minimizing the pumping losses at low loads and mitigating the knock occurrence at high loads [37]. In the medium BMEP range, especially for strategy \#2, IVC is slightly delayed, allowing for higher air flow rates and resulting in a better agreement with the Opt outcomes. EVC indeed exhibits an approximately constant trend with load. 


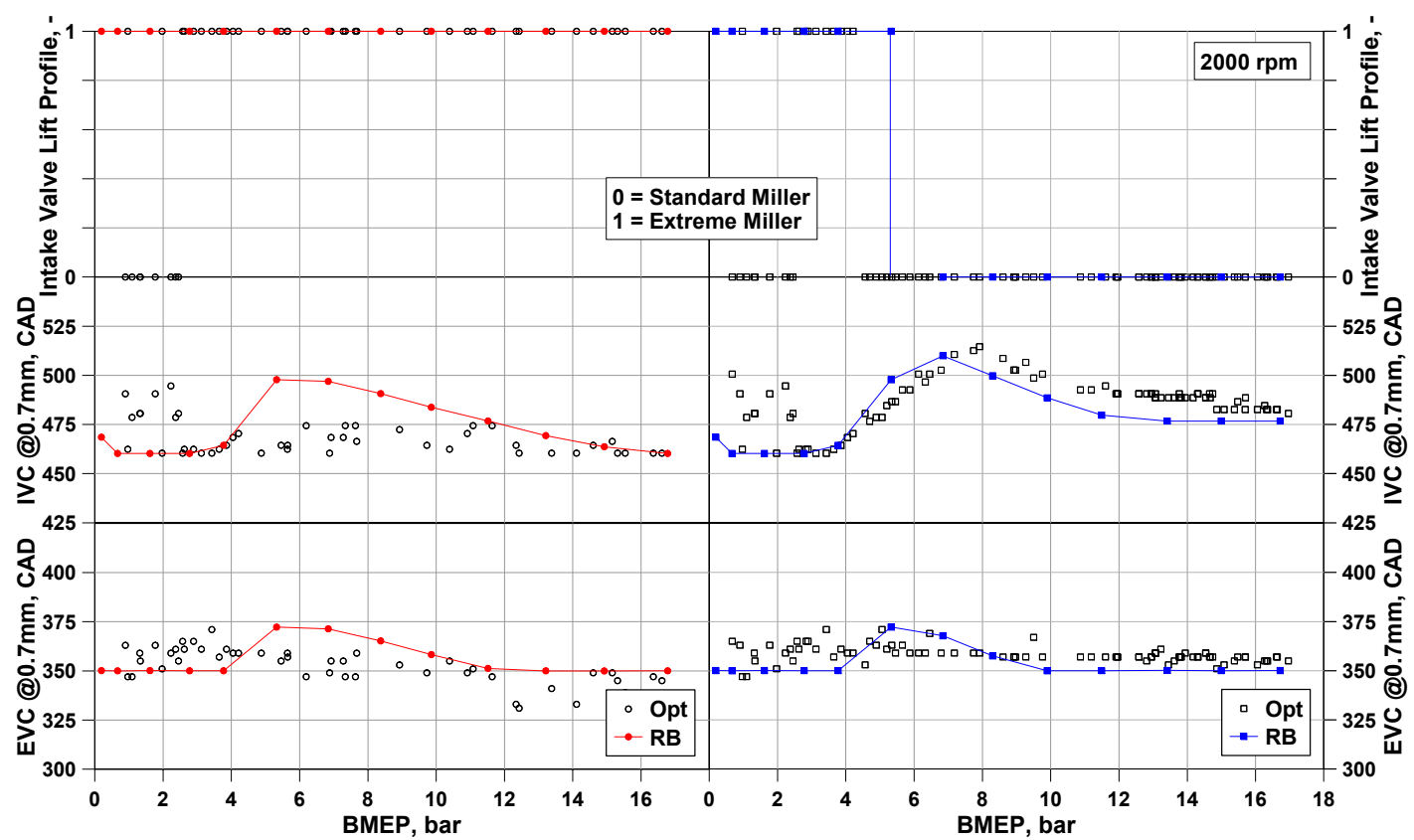

(a) strategy \#1

(b) strategy \#2

Figure 5. Selected intake valve lift profile, IVC and EVC, comparison in a BMEP sweep at $2000 \mathrm{rpm}$ for strategies \#1 (a) and \#2 (b).

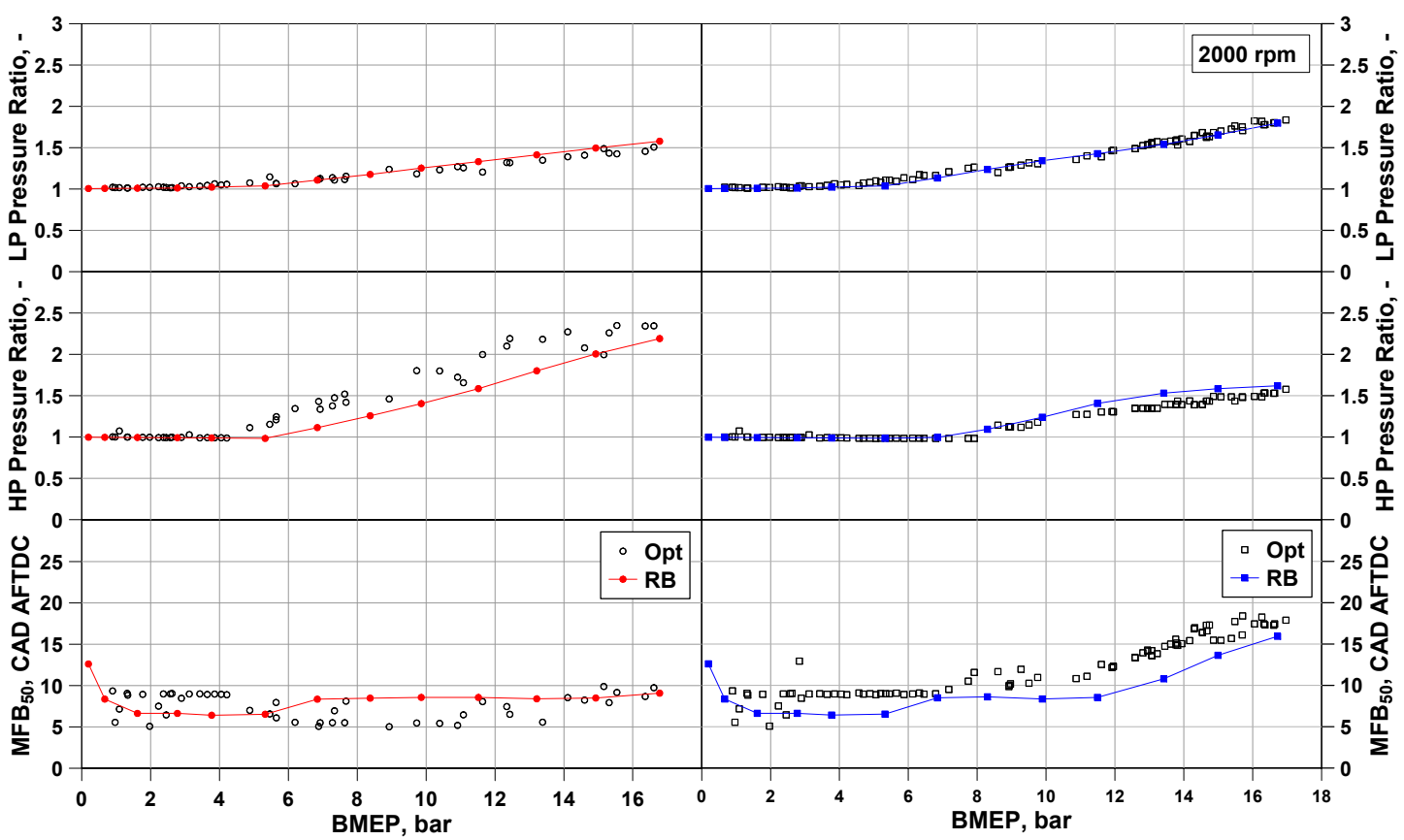

(a) strategy \#1

(b) strategy \#2

Figure 6. Low/High pressure ratios and $\mathrm{MFB}_{50}$ comparison in a BMEP sweep at $2000 \mathrm{rpm}$ for strategies \#1 (a) and \#2 (b).

Figure 6 shows the sharing of the overall engine boosting between the two compressors, depicting the HP and the LP pressure ratios. As expected, strategy \#1 favored a higher boosting by the E-Comp instead of the LPC. Conversely, for strategy \#2 the boosting was principally realized by the LPC; instead, the E-Comp worked by having a pressure ratio as low as possible. This choice involved an increased engine exhaust backpressure, but it resulted in a lower power absorbed by the E-Comp. 
The results in Figure 6 also show that HP and LP pressure ratios assume unit values below 5 bar BMEP, suggesting a throttle-based load control. Above 5 bar, both LP and HP boosting are progressively regulated by a partial closing of the LPT rack and by simultaneously increasing the E-Comp power.

It is worth underlining that the RB strategy \#1 between 4-10 bar shows some discrepancies if compared to the optimal outcomes. More specifically, the RB approach determined a delayed IVC, which is compensated by a lower HP boost pressure. Nevertheless, this reflects a slight BTE lowering compared to the optimizer output (less than $1 \%$ ).

As a further assessment between RB and Opt methodologies, in Figure 6 the BMEP trends of $\mathrm{MFB}_{50}$ are reported, highlighting a satisfactory RB/Opt agreement. For this combustion parameter, the optimal value is usually around 6-10 CAD AFTDC. Indeed, in the case of later phasing, the combustion develops during the expansion stroke, leading to a less effective work exchange between the piston and the gas, while, in the case of too advanced $\mathrm{MFB}_{50}$, a relevant portion of the combustion process takes place during the compression stroke, increasing the compression work exerted by the piston on the fluid. An almost flat trend was obtained for strategy \#1 in a wide load range (2-15 bar BMEP) with most of the points in the band 5-10 CAD AFTDC. A slightly delayed MFB 50 at both higher and lower BMEP values was detected. At high loads, this is due to knock mitigation, while at low loads, it is because of the combustion lengthening and of the limitation on the maximum allowable spark advance. For the strategy \#2, $\mathrm{MFB}_{50}$ showed a different trend with a more pronounced delay of the $\mathrm{MFB}_{50}$ at increasing loads (above 10 bar BMEP). This, of course, is the only possibility for knock control once the Standard Miller profile for the intake valves has been selected. This quite different behavior was very well captured by the RB strategies.

Although not discussed here for sake of brevity, another outcome of the optimization is that a close-to-stoichiometric mixture is preferred in the pre-chamber for whatever is the BMEP. A certain mixture leaning is required at high loads to limit the pressure peak in the PC.

To further prove the consistency of the RB calibration, Figures 7 and 8 present additional comparisons between RB and Opt strategies, referring to the engine speed of $3000 \mathrm{rpm}$. Once again, a satisfactory RB/Opt agreement for all the considered variables was reached all along the BMEP sweep. Figure 7 shows that, for this higher speed, the optimal control involved more relevant modifications of intake valve lift profiles and IVC/EVC timings at changing loads.

For strategy \#1, different from the previous speed, the Standard Miller was selected in a restricted low/medium load range (4-5.5 bar BMEP) to enhance the effective compression ratio and hence the efficiency in the light of a minor knock tendency at this speed. However, the Extreme Miller was once again preferred as soon as the load increases (Figure 7). For strategy \#2, an earlier transition from the Extreme Miller to the Standard Miller by increasing the BMEP was detected if compared to the results at $2000 \mathrm{rpm}$. Once again, at medium loads the IVC was delayed, allowing for increased airflow rates. EVC trends appear almost load insensitive regardless of the calibration strategy, and a modest discrepancy between RB and Opt procedures was detected especially at very low BMEPs (0-2 bar in Figure 7). This is probably due to a more refined calibration given by the optimizer, which tends to reduce the valve overlap with the aim to limit the internal EGR even more than the logics of the RB approach. Referring to the results plotted in Figure 8, similar considerations to the ones already carried out for the case at $2000 \mathrm{rpm}$ still hold.

Concerning the combustion phasing differences between 2000 and $3000 \mathrm{rpm}$, a greater delayed $\mathrm{MFB}_{50}$ was observed at low loads for the engine speed of $3000 \mathrm{rpm}$, mainly ascribed to a pronounced combustion lengthening of its initial stage due to an excessive rich mixture in the pre-chamber at the spark event. This is due to the lower bound of the PC injection duration of $300 \mu$ s (see Table 1). At high loads, a less pronounced $\mathrm{MFB}_{50}$ delay occurred for the strategy \#2 due to the lower knock tendency at $3000 \mathrm{rpm}$. In summary, the above discussed results highlight that the RB calibration demonstrates to be extremely trustworthy for various load levels and for two different speeds, allowing to reach efficiencies and control variables very close to the ones obtained by the optimizer-based methodology. In the light of the latter consideration, the developed RB calibration was broadened to the full engine speed/load 
range in order to investigate the close-to-optimal engine performance in the whole operating domain, as reported hereinafter.

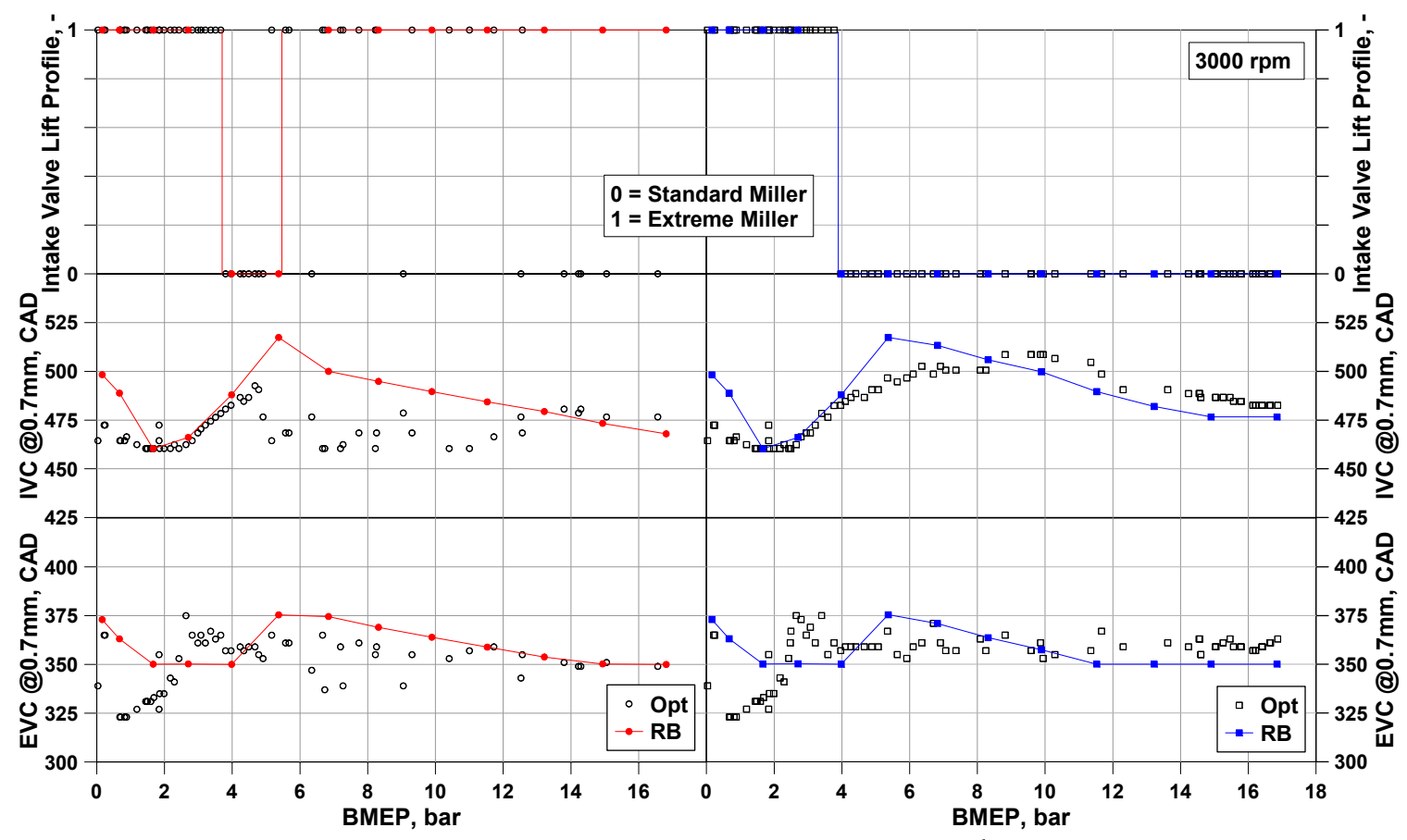

(a) strategy \#1

(b) strategy \#2

Figure 7. Intake valve lift profile, IVC, and EVC comparison in a BMEP sweep at $3000 \mathrm{rpm}$ for strategies \#1 (a) and \#2 (b).

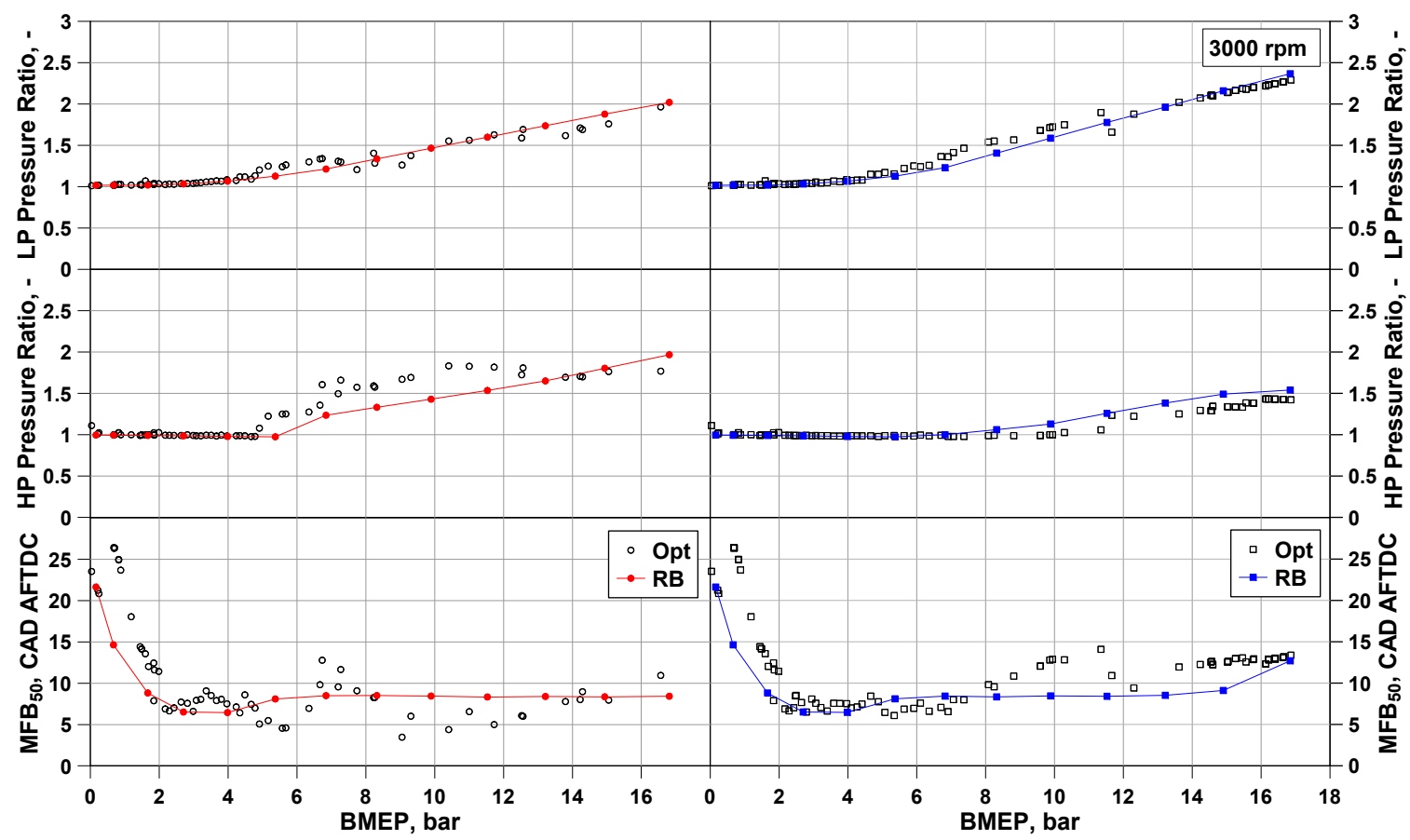

(a) strategy \#1

(b) strategy \#2

Figure 8. Low/High pressure ratios and $\mathrm{MFB}_{50}$ comparison in a BMEP sweep at $3000 \mathrm{rpm}$ for strategies $\# 1$ (a) and \#2 (b). 


\section{Map of Engine Performance}

According to the RB control strategy, the entire engine operating map was computed (consisting in 143 operating points $-11 \mathrm{rpm} \times 13 \mathrm{BMEP}$ ) for the two different calibration strategies above discussed. In Figure 9, the BTE maps for calibration strategies \#1 (a) and \#2 (b) are shown. The maximum levels for both strategies occur at $2000 \mathrm{rpm}$, close to the full load region. The OBTE maps for the different strategies are shown in Figure 10. For these, the maxima occur at medium speeds (2000-2500 rpm) and medium-high loads (8-14 bar BMEP). According to the selected optimization targets, a $3 \%$ higher peak BTE was obtained in the strategy \#1 (Figure 9a), while a 1\% higher peak OBTE was obtained in the strategy \#2 (Figure 10b).

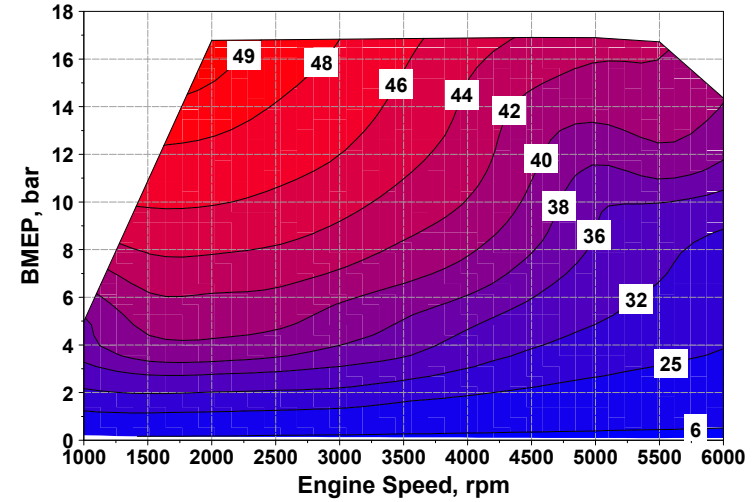

(a) BTE strategy \#1

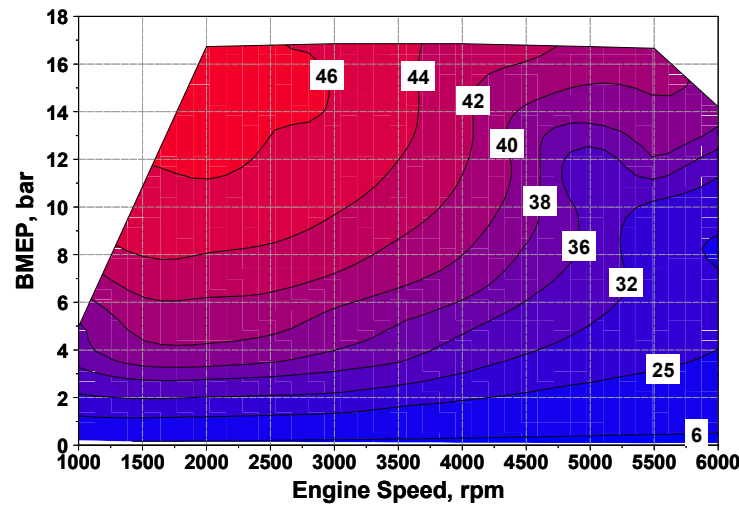

(b) BTE strategy \#2

Figure 9. Map of BTE (\%) for strategies \#1 (a) and \#2 (b).

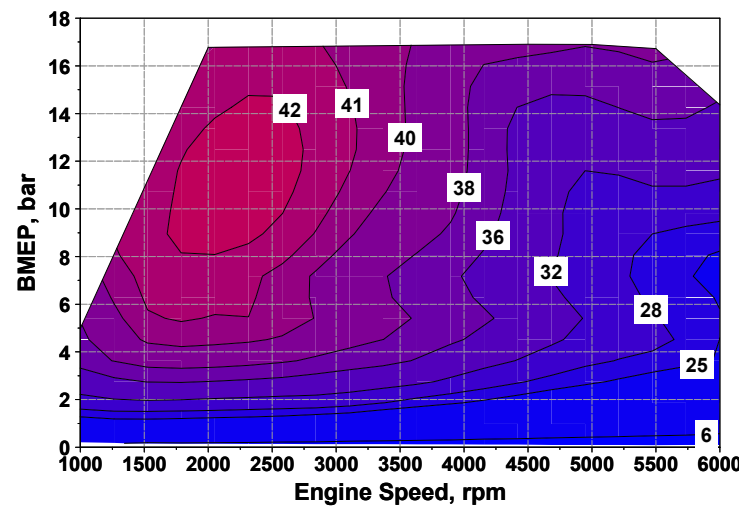

(a) OBTE strategy \#1

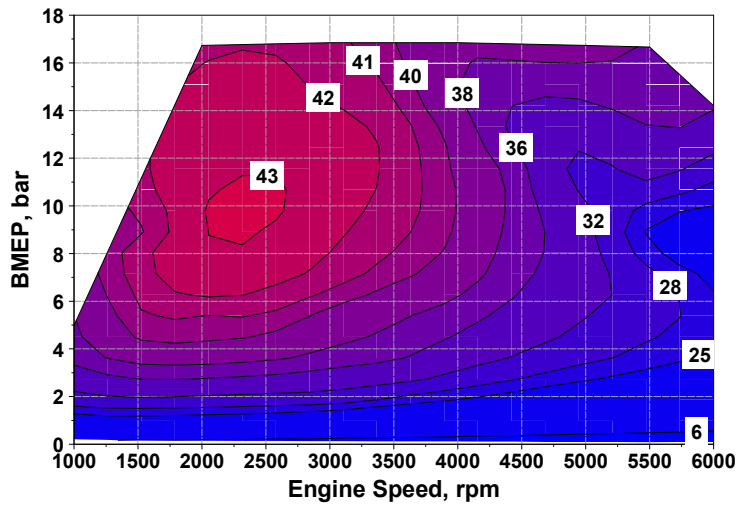

(b) OBTE strategy \#2

Figure 10. Map of OBTE (\%) for strategies \#1 (a) and \#2 (b).

The gap between BTE and OBTE for the strategy \#1 was about 7\% in the OBTE peak zone, and gradually decreased at higher speeds and low loads. A similar behavior characterizes the strategy \#2, having in the peak zone a gap of about 3-4\%. This is due to the power absorbed by the E-Comp, whose maps are reported in Figure 11. For strategy \#1, the E-Comp power gets to the upper bound of $10 \mathrm{~kW}$, which is about the $8 \%$ of the power rated by the engine, while for strategy \#2, it only gets a maximum of about $7 \mathrm{~kW}$. 


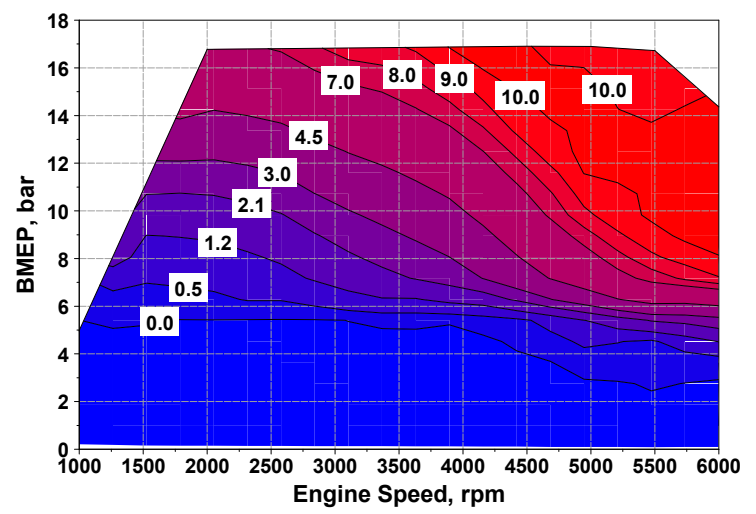

(a) E-Comp Power, strategy \#1

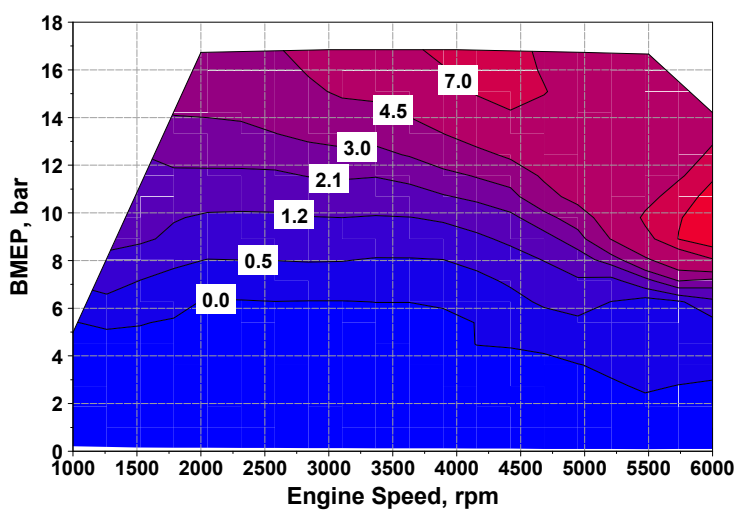

(b) E-Comp Power, strategy \#2

Figure 11. Map of E-Comp power consumption $(\mathrm{kW})$ for strategies \#1 (a) and \#2 (b).

For the considered engine, featuring an ultra-lean air/fuel mixture, both strategies involved a very high plenum pressure, which is mandatory to achieve the load target. As an example, Figure 12a shows the boost pressure map for the strategy \#1, which highlights a peak of 4.3 bar at the highest speeds and loads. The intake plenum pressure was conserved above 1.0 bar in most of the map (reduced pumping losses), and above 0.5 bar even in the area at lowest BMEP. The lambda target of 2 in the main chamber was reached in most of the operating domain, as shown in Figure 12b, except in the maximum power area. This is due to the attainment of the maximum E-Comp power, which obliges to reduce the mixture leaning $\left(\lambda_{\mathrm{MC}}<2\right)$ to get the load. Similar boost pressure and lambda maps are also found for the strategy \#2.

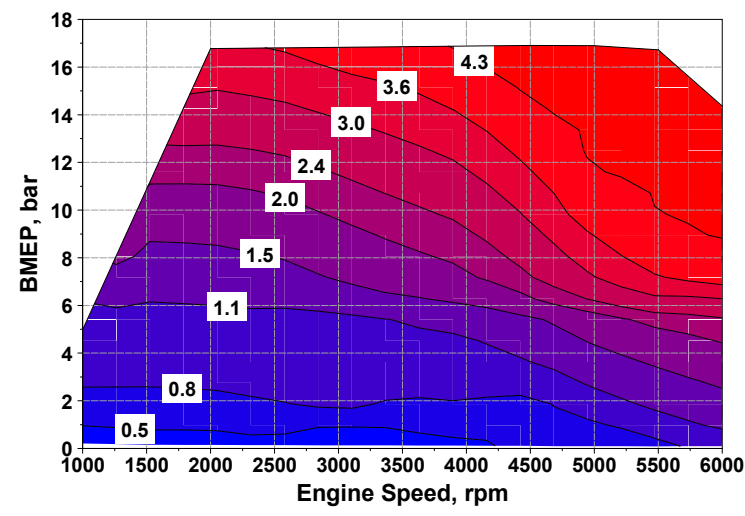

(a) Intake plenum pressure, strategy \#1

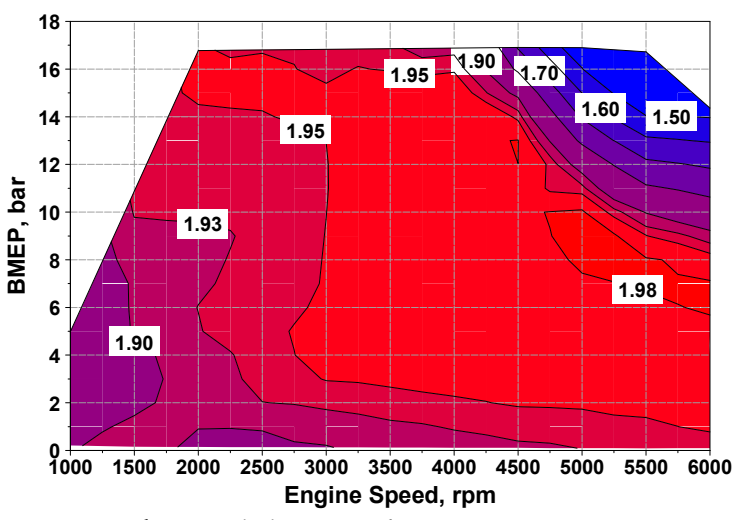

(b) Lambda ratio, $\lambda_{\mathrm{MC}}$, strategy $\# 1$

Figure 12. Map of intake plenum pressure (bar) (a) and (b) map of $\lambda_{\mathrm{MC}}$ (-) for strategy \#1.

The predicted brake-specific emissions of $\mathrm{NO}_{\mathrm{x}}$ and $\mathrm{HC}$ are shown in Figure 13a,b, respectively, still referring to strategy \#1. With the aim to limit the temperature peaks in the burned zone of the combustion chamber preventing the formation of the above pollutants, the ultra-lean combustion concept was used. The $\mathrm{NO}_{\mathrm{x}}$ production was very low in most parts of the operating map, except for the high speed and load zone. This is due to the lower mixture leaning in the above-mentioned zone, compared to the $\lambda_{\mathrm{MC}}=2$ target (see $\lambda_{\mathrm{MC}}$ contours in Figure 12b). Due to the higher burned temperature, if compared to the one in the $\mathrm{MC}$, for this engine the $\mathrm{NO}_{\mathrm{x}}$ production was prevailing in the pre-chamber. Despite the large excess air, the HC production is not negligible over the whole engine domain (Figure 13b). This is due to the lean burning concept, which, compared to a conventional stoichiometric engine, reduces the in-cylinder temperatures and consequently makes the post-oxidation phenomenon less effective. Looking to Figure 13b, the shape of the HC iso-contours is justified by a faster post-oxidation at increasing speed (less time for wall heat losses) and load (lower percent wall heat losses). 


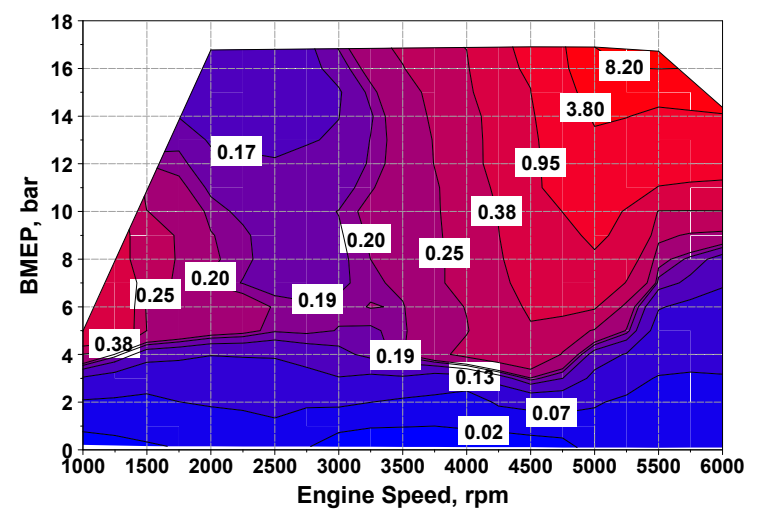

(a) BSNOx, strategy \#1

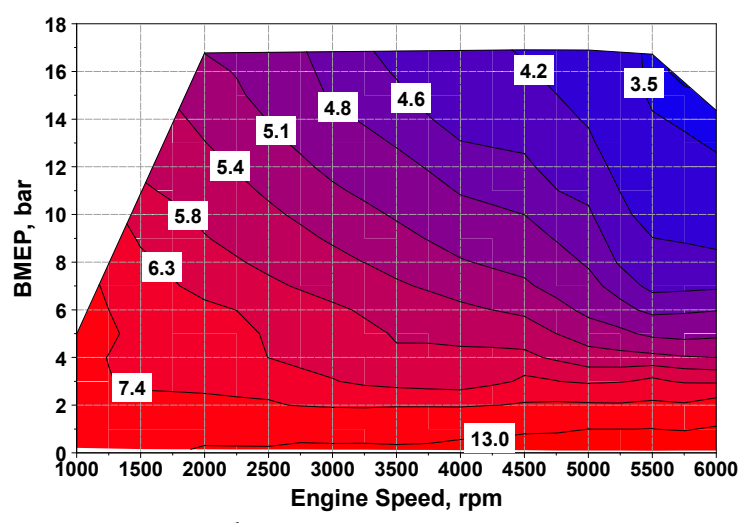

(b) BSHC, strategy \#1

Figure 13. Map of (a) brake specific $\mathrm{NO}_{\mathrm{x}}$ emission $(\mathrm{g} / \mathrm{kWh})$ and (b) map of brake-specific $\mathrm{HC}$ emission $(\mathrm{g} / \mathrm{kWh})$ for strategy \#1.

\section{Vehicle Simulations}

\subsection{Hybrid Vehicle Architecture}

The main characteristics of the vehicle here investigated are reported in Table 2 [38]. The vehicle is a HEV, belonging to the C-class. It is characterized by a combined parallel/series powertrain, schematized in Figure 14. The powertrain is composed of an ICE, two electric motor/generator units, (EM and EG), a battery $(\mathrm{Ba})$, three clutches $\left(\mathrm{Cl}_{1-3}\right)$ and two gear boxes $\left(\mathrm{GB}_{1-2}\right)$.

Table 2. Main characteristics of the tested HEV.

\begin{tabular}{|c|c|}
\hline \multicolumn{2}{|l|}{ Vehicle } \\
\hline Mass, kg & 1730 \\
\hline Car aero drag, $\mathrm{m}^{2}$ & 0.775 \\
\hline Tire rolling resistance coeff., - & 0.008 \\
\hline Wheel diameter, $\mathrm{m}$ & 0.723 \\
\hline Axle ratio, - & 4.4 \\
\hline Axle inertia, $\mathrm{kgm}^{2}$ & 1.5 \\
\hline \multicolumn{2}{|c|}{ Internal Combustion Engine } \\
\hline Displacement, $\mathrm{cm}^{3}$ & 1633.1 \\
\hline Max Power, kW & 125 \\
\hline Inertia, $\mathrm{kgm}^{2}$ & 0.35 \\
\hline \multicolumn{2}{|l|}{ Electric Generator } \\
\hline Max Power, kW & 55 \\
\hline Max Torque, Nm & 165 \\
\hline Inertia, $\mathrm{kgm}^{2}$ & 0.10 \\
\hline \multicolumn{2}{|l|}{ Electric Motor } \\
\hline Max Power, kW & 50 \\
\hline Max Torque, Nm & 240 \\
\hline Inertia, $\mathrm{kgm}^{2}$ & 0.10 \\
\hline \multicolumn{2}{|l|}{ Battery } \\
\hline Internal Resistance, Ohm & 0.375 \\
\hline Voltage, Volt & 400.0 \\
\hline Energy density, Wh/kg & 170.0 \\
\hline Usable battery sizing, $\mathrm{kWh}$ & 0.50 \\
\hline SoC limits, - & $0.2-0.9$ \\
\hline
\end{tabular}


Table 2. Cont.

\begin{tabular}{cc}
\hline \multicolumn{2}{c}{ Gear-Box 1} \\
\hline Gear 1 Ratio, - & 2.72 \\
Gear 2 Ratio, - & 1.64 \\
Gear 3 Ratio, - & 0.99 \\
Gear 4 Ratio, - & 0.60 \\
\hline \multicolumn{2}{c}{ Gear-Box 2} \\
\hline Gear 1 Ratio, - & 2.67 \\
Gear 2 Ratio, - & 1.03 \\
\hline
\end{tabular}

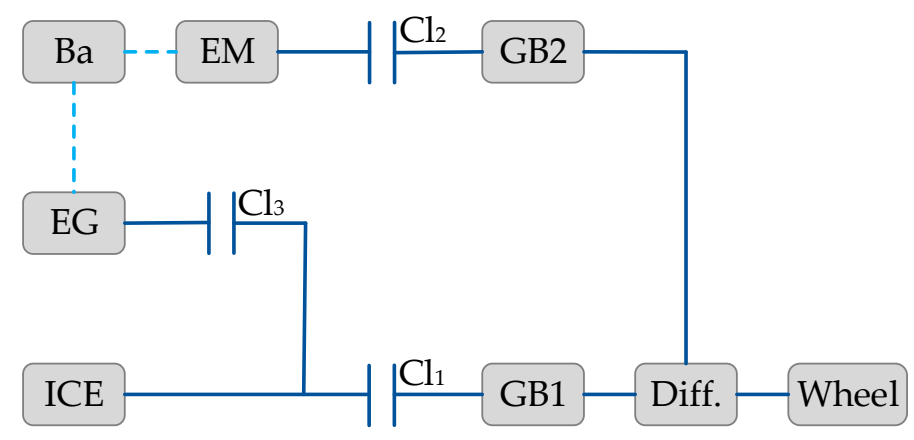

Figure 14. Powertrain schematic of the tested HEV.

\subsection{Simulation Platform}

The vehicle simulations were carried out by a "in-house developed" software, implemented in Fortran language (UniNa Vehicle Simulation-UNVS) [21]. It is based on a "backward-facing" (quasi-static) method [39]. All of the powertrain schematic components in Figure 14 were defined by a lumped-parameter method. The approach to describe the thermal unit was quasi-steady map based. In particular, the BSFC maps, derived from the calibration strategies \#1 and \#2, were implemented in the vehicle simulation, together with the curve of maximum shaft torque. In addition to the BSFC maps, the vehicle model handled the corresponding E-Comp power consumption maps. The electrical absorption of the E-Comp is considered as an additional load on the battery. The ICE thermal transient was neglected in the simulations, resulting in a null fuel consumption penalization at the cold start. The exhaust gas after-treatment system was not modeled, so the predicted emissions have to be considered as "engine-out" values. The electric unit efficiencies were described once again by a map-based approach, dependent on speed and shaft torque. The battery was treated by a conventional State of Charge (SoC) model [40]. In order to estimate the Joule-effect losses, an internal resistance was set, neglecting the possible variations in changing SoC and temperature. The mechanical losses in the gearboxes were evaluated assuming constant efficiencies (equal to 0.97).

\subsection{Efficient Thermal Electric Skipping Strategy}

The vehicle control strategy here adopted, if compared to the well assessed strategies (namely Pontryagin minimum principle [41], dynamic programming [42] or ECMS [43]), is designed to be simplified. The conventional power-split logics, typical of the abovementioned strategies, is substituted with an alternative utilization of the thermal and electric units for the vehicle driving (Efficient Thermal Electric Skipping Strategy-ETESS [21]). The basic concept behind ETESS is an alternate use of the electric units and thermal engine so to fulfill the power demand at the vehicle wheels. The choice between the two modes depends, at each time, on the comparison between the effective fuel rate of the thermal engine needed to fully satisfy the power demand at the vehicle wheels, and an equivalent fuel rate related to a pure electric driving of the vehicle. The ETESS is featured by a 
much-reduced computational effort if compared to most common optimization strategies, leading to similar performance in terms of kilometric consumption and emission [21]. The results shown in the next section were obtained by the off-line variant of the ETESS, where the control is tuned to realize the battery energy balance between the beginning and the end of the driving mission [44].

\subsection{Simualtion Raster and Results Discussion}

The vehicle simulations were carried out for two driving cycles, namely the WLTC and a real driving cycle (RDC) [45]. The results of $\mathrm{CO}_{2}$ and pollutant emissions (grams per kilometer) are represented under the form of bar charts in Figures 15 and 16. Each couple of bars compares the results of the calibration strategy \#1 (red) and strategy \#2 (blue), reporting on the top percent difference.

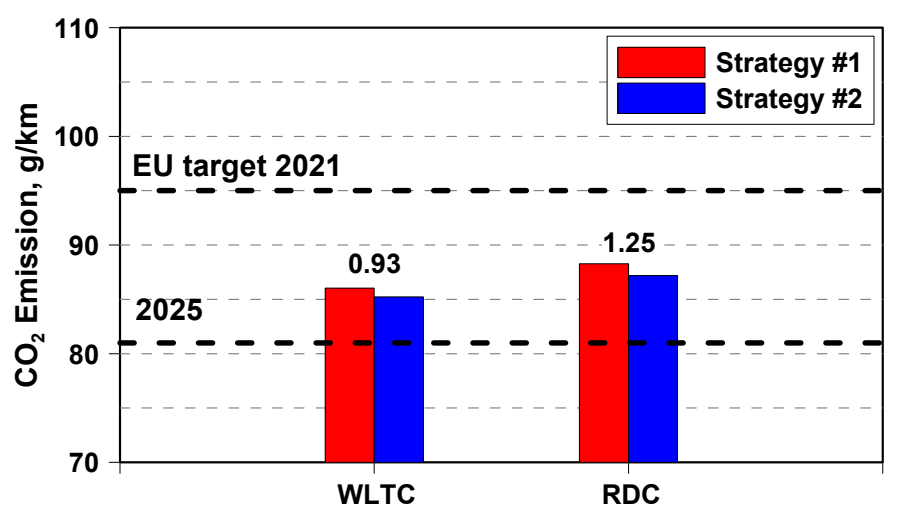

Figure 15. Strategies assessment of $\mathrm{CO}_{2}$ emission and their percent difference along WLTC and RDC.

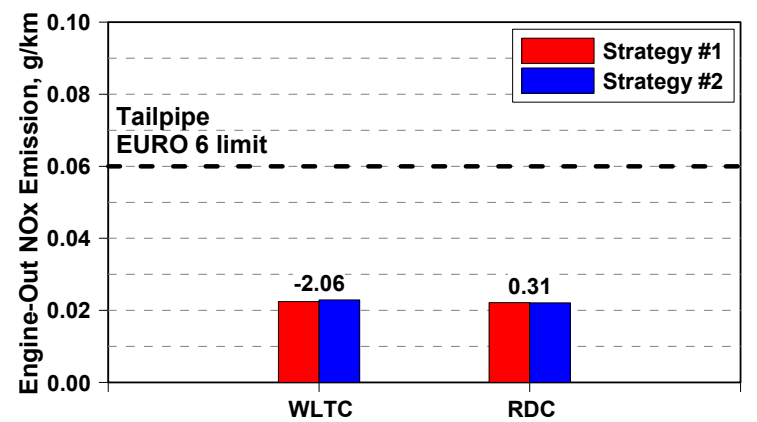

(a)

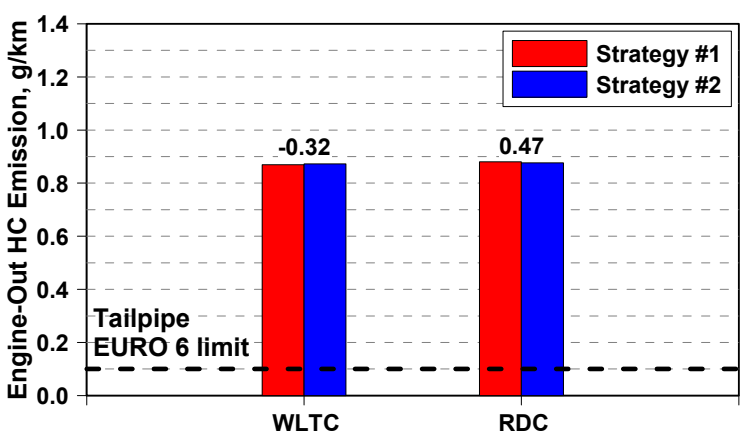

(b)

Figure 16. Strategies assessment of $\mathrm{NO}_{\mathrm{x}}$ emission (a) and of $\mathrm{HC}$ emission (b) and their percent difference along WLTC and RDC.

Starting from the $\mathrm{CO}_{2}$ results shown in Figure 15, a first consideration is that the emissions are below the target imposed by the European Union for the 2021 of $95 \mathrm{~g} / \mathrm{km}$, but above the one of the 2025 , equal to about $81 \mathrm{~g} / \mathrm{km}$ or whatever is the ICE control strategy. These results indicate that the HEV vehicle here analyzed, even equipped with a very efficient thermal unit, was not enough to match the EU 2025 target and a plug-in HEV variant is mandatory to get this. The pollutant emission outcomes, as stated above, refer to engine-out values compared to the tailpipe reference standards imposed by the legislation. The potential abatement due to an aftertreatment system is not explicitly considered. However, the comparison can also help to evaluate and design the most adequate exhaust after-treatment system (EATS) for the considered application. Looking at the values for the nitrogen oxides (Figure 16a), it can be seen that the emissions are within the bounds. The HC values (Figure 16b), for both the considered cycles and calibration strategies, are much higher than the limit imposed from the Euro 6d, highlighting the need for an oxidizing EATS for regulation compliance. Anyway, the technology of an oxidizing EATS at the current state-of-art is very robust, low-cost and reliable, 
not representing a substantial challenge for a real on-vehicle application. The engine calibration, either strategy \#1 or \#2, does not seem to exert a substantial influence on the pollutant emissions. It is worth noting that the ETESS control strategy was not conceived to minimize the pollutant emissions, but the fuel consumption and hence the $\mathrm{CO}_{2}$ emissions.

The comparison presented underlines that the strategy \#2 allows slightly lower $\mathrm{CO}_{2}$ emissions for both the considered driving cycles. To clarify this result, the instantaneous trends of some representative control and performance parameters are plotted in Figure 17 with reference to the WLTC. The trends underline that the powertrain control is practically the same for both the ICE calibrations, resulting in almost superimposed traces of ICE power (b), EM power (c) and gear number (g). Some differences emerge for the trends of E-Comp power (d) and SoC (e), which reflect slight misalignments of fuel consumption (f) and battery power (h) profiles. Note that a positive value of battery power corresponds to an energy flux drained from the battery.

To better highlight the differences among the two engine calibrations, the plots of SoC, fuel rate and battery power are reproposed in Figure 18 with a magnified time scale in the most load-demanding portion of the driving cycle, namely between 1450-1750 s. During this period, the fuel rate is lower for the calibration strategy \#1 if compared to the strategy \#2, while the opposite occurs for the battery power consumption due to a higher E-Comp power absorption (Figure 17d). This reflects in a faster decrease of the SoC, which leads to reduced electric driving in the first portion of the cycle where the load demand is lower. For this reason, the ICE works for a longer time in the operating conditions, namely at low load/speed, where its efficiency is well below its maximum level. On the contrary, the calibration strategy \#2 allows longer pure electric driving in the first portion of the cycle, avoiding for the thermal engine operations with a very reduced efficiency.

A similar behavior emerged for the RDC, as shown in Figure 19. This reports the vehicle speed profile (a) and the SoC trend (b). Note that the initial SoC level was chosen to get simultaneously the optimal control and battery energy balance along the cycle. Such a choice is a consequence of the simplified approach for the battery treatment, where the losses are not dependent on the instantaneous SoC level and on the possible stress when the extreme SoC bounds are attained.

Once again, during the most load-demanding portion of the cycle (after about $5400 \mathrm{~s}$ ), the battery discharge for the calibration strategy \#1 was faster, and this was compensated by a reduced use of electric driving in the first portion of the cycle. This occurrence globally penalizes the strategy \#1 in comparison with the strategy \#2.

Under a more general point of view, for a given battery capacity and vehicle mission, the engine calibration, which reduces the energy drain from the battery, allows for a more efficient choice of the electric driving phase along the cycle. In the presented cases, however, a limited benefit has been estimated. An explanation of such reduced fuel consumption differences can be drawn by analyzing the time evolution of the engine operating point. Referring to the RDC, the instantaneous engine operating points, sampled at each second, are shown in Figure 20. They are plotted over the map of OBTE difference between strategies \#2 and \#1. It can be observed that most of the samples are located in a speed range between 1500 and $2500 \mathrm{rpm}$ and with loads below 8/9 bar BMEP, where the OBTE difference is lower than $0.6 \%$ and the E-Comp power is small (Figure 11). Changes between the two calibrations only emerge outside the above operating area where the percentage difference in terms of OBTE increases, contributing to reduce the fuel consumption of strategy \#2. Similar considerations can be applied to the WLTC results. Different driving missions and more load demanding are expected to bring out more relevant differences between the considered strategies. 

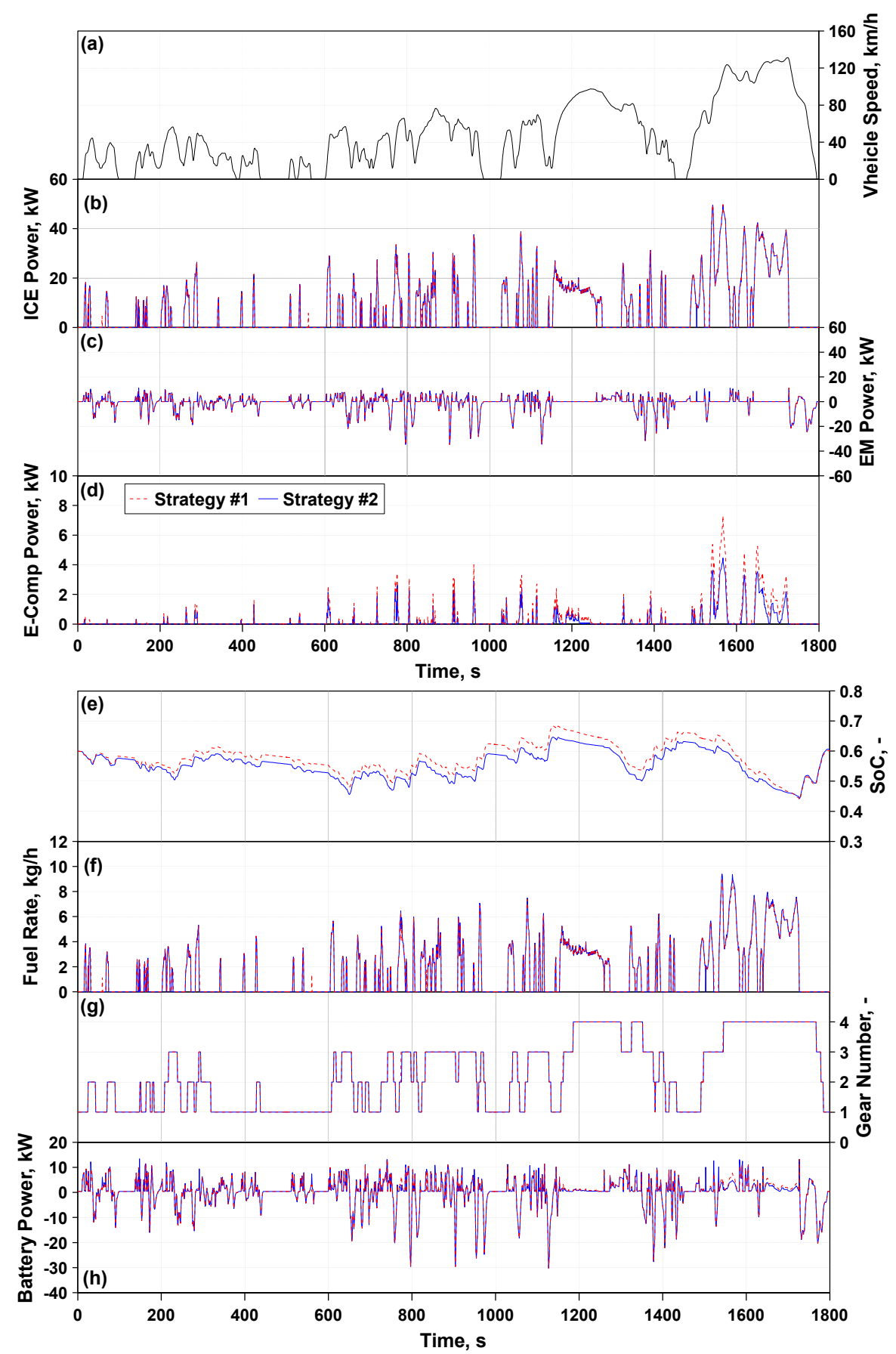

Figure 17. Instantaneous trends of vehicle speed (a), ICE power (b), EM power (c), E-Comp power (d), SoC (e), fuel rate (f), gear number $(\mathbf{g})$, and battery power $(\mathbf{h})$ along WLTC.

Even if the advantage of the strategy \#2 cannot be considered yet as a general result due to the need of further verifications with different powertrain/vehicle types, it seems that a reduced battery consumption, even if the thermal engine will not give its maximum efficiency performance, is the path to improve the hybrid vehicle fuel economy. As stated above, further analyses will be performed in the next development of this activity to confirm the generality of this result. 


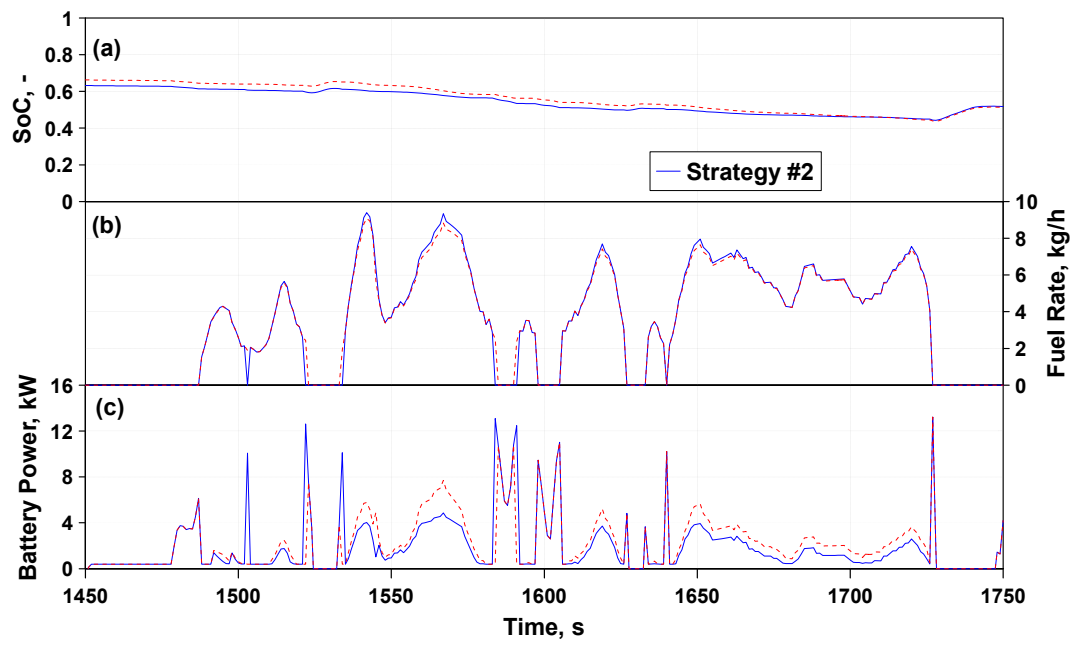

Figure 18. Instantaneous trends of $\operatorname{SoC}(\mathbf{a})$, fuel rate (b), and battery power (c) along the last portion of WLTC.

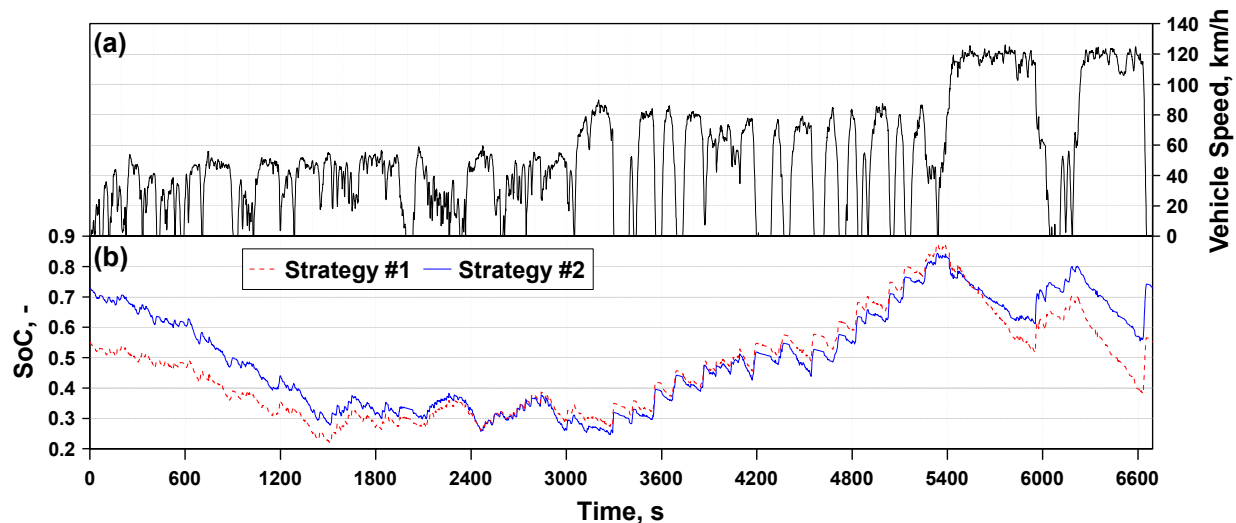

Figure 19. Instantaneous trends vehicle speed (a) and SoC (b) along the RDC.

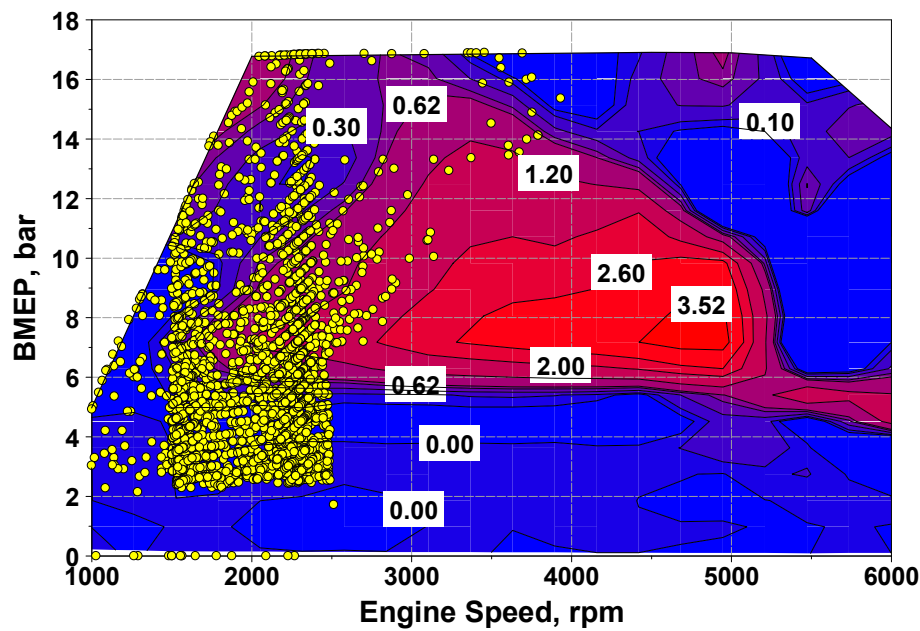

Figure 20. Instantaneous engine operating point along the RDC over the OBTE difference between strategy \#2 and \#1.

\section{Conclusions}

In this paper, a refined study of an innovative engine prototype was provided, aiming to estimate the impact of the engine calibration on $\mathrm{CO}_{2}$ and pollutant emission of a hybrid vehicle equipped with the considered power unit, consisting in a two-stage boosted ultra-lean pre-chamber SI engine. 
As a first step, a physical model for the prediction of the burn rate for this engine architecture was recalled, able to sense its main geometrical parameters and operating conditions. The combustion model, validated in previous authors' activities, was integrated with advanced sub-models of turbulence, heat transfer, knock, and engine-out pollutant emissions formation.

The model was employed to estimate complete engine performance maps once a rule-based numerical pre-calibration had been set up. In particular, two variants of the calibration procedure were proposed, aiming to maximize either the BTE or the OBTE; this last option also considered the E-Comp power consumption. Despite the great number of control parameters, the RB approach was successfully verified against the results of a more complex and time-consuming procedure where the 1D engine model was coupled to an external optimizer built on a genetic algorithm (MOGA-II).

The estimated performance maps revealed the potential of this advanced engine architecture to reach very high efficiency levels. Depending on the calibration strategy, a 49\% BTE peak was estimated at $2000 \mathrm{rpm}$ (full load), and a 43\% OBTE peak was also gained at a lower load. The analyses also underline low cylinder-out specific $\mathrm{NO}_{x}$ emissions, thanks to the ultra-lean combustion concept and relevant cylinder-out specific HC emissions, due to an ineffective post-oxidation process.

The computed engine performance maps were employed in vehicle simulations to assess these strategies along two representative driving cycles, namely WLTC and an RDC. The comparisons underline that the strategy \#2 allows slightly lower $\mathrm{CO}_{2}$ emissions for both the considered driving cycles. This is due to a more extended pure electric driving during the low-speed portion of the driving cycles, allowed by a reduced electric consumption in the high-speed driving mission. In this way, the time in which the engine operates with a reduced efficiency was shortened. Either control strategies do not substantially show a clear impact on the pollutant emissions.

The discussed methodology represents a potential powerful tool to forecast both engine and vehicle performance. Once tested and validated against experimental data, this procedure will contribute to supporting and driving the development phase of a high-efficient HEV.

Author Contributions: Conceptualization, F.B. and V.D.B.; Methodology, F.B. and V.D.B.; Software, E.M.; Validation, E.M., L.T. and D.T.; Data Curation, E.M., L.T. and D.T.; Writing_Original Draft Preparation, F.B., V.D.B., E.M., L.T. and D.T.; Writing-Review \& Editing, V.D.B. and E.M.; Visualization, E.M., L.T. and D.T.; Supervision, F.B. and V.D.B.; Project Administration, V.D.B.; Funding Acquisition, F.B. and V.D.B. All authors have read and agreed to the published version of the manuscript.

Funding: This project has received funding from the European Union's Horizon 2020 research and innovation programme under grant agreement No 724084.

Conflicts of Interest: The authors declare no conflict of interest.

\section{Definitions/Abbreviations}

$\begin{array}{ll}\text { 0D/1D } & \text { Zero/One Dimensional } \\ \text { AFTDC } & \text { After Top Dead Center } \\ \text { AI } & \text { Auto Ignition } \\ \text { Ba } & \text { Battery } \\ \text { BB } & \text { Base Boost } \\ \text { BEV } & \text { Battery Electric Vehicles } \\ \text { BMEP } & \text { Brake Mean Effective Pressure } \\ \text { BSFC } & \text { Brake Specific Fuel Consumption } \\ \text { BSHC } & \text { Brake Specific HC } \\ \text { BSNO } & \text { Brake Specific NOX } \\ \text { BTDC } & \text { Before Top Dead Center } \\ \text { BTE } & \text { Brake Thermal Efficiency } \\ \text { CAD } & \text { Crank Angle Degree } \\ \text { Cl } & \text { Clutch } \\ \text { DI } & \text { Direct Injection } \\ \text { DP } & \text { Dynamic Programming } \\ \text { EATS } & \text { Exhaust After-Treatment System }\end{array}$




\begin{tabular}{|c|c|}
\hline EG & Electric Generator \\
\hline EGR & Exhaust Gas Recirculation \\
\hline EM & Electric Motor \\
\hline EML & Extreme Miller Lift \\
\hline ETESS & Efficient Thermal Electric Skipping Strategy \\
\hline EVC & Exhaust Valve Closure \\
\hline EU & European Union \\
\hline FL & Full Load \\
\hline GB & Gear Box \\
\hline $\mathrm{HCCI}$ & Homogeneous Charge Compression Ignition \\
\hline $\mathrm{HEV}$ & Hybrid Electric Vehicle \\
\hline $\mathrm{HP}$ & High-Pressure \\
\hline HPC & High-Pressure Compressor \\
\hline ICE & Internal Combustion Engine \\
\hline IVC & Intake Valve Closure \\
\hline LHV & Low Heating Value \\
\hline LP & Low-Pressure \\
\hline LPC & Low-Pressure Compressor \\
\hline LPT & Low-Pressure Turbine \\
\hline MC & Main Chamber \\
\hline MFB & Mass Fraction Burned \\
\hline OBTE & Overall Brake Thermal Efficiency \\
\hline PC & Pre Chamber \\
\hline PFI & Port Fuel Injection \\
\hline PHEV & Plug-in Hybrid Electric Vehicle \\
\hline PID & Proportional Integral Derivative \\
\hline PMP & Pontryagin Minimum Principle \\
\hline $\mathrm{RB}$ & Rule Based \\
\hline $\mathrm{RDC}$ & Real Driving Cycle \\
\hline RDE & Real Driving Emission \\
\hline SACI & Spark Assisted Compression Ignition \\
\hline SI & Spark Ignition \\
\hline SML & Standard Miller Lift \\
\hline SoC & State of Charge \\
\hline UNVS & UniNa Vehicle Simulation \\
\hline VVT & Variable Valve Timing \\
\hline WLTC & Worldwide harmonized Light vehicles Test C \\
\hline
\end{tabular}

\section{Symbols}

$\begin{array}{ll}\mathrm{A} & \text { Area } \\ A_{L} & \text { Laminar Flame Area } \\ c_{j e t} & \text { Tuning constant } \\ \mathrm{k} & \text { Turbulent kinetic energy } \\ \mathrm{K} & \text { Mean flow kinetic energy } \\ m_{b} & \text { Burned mass } \\ m_{b, e n t r} & \text { Burned portion of entrained mass } \\ m_{\text {entr }} & \text { Current entrained mass } \\ \dot{m}_{f} & \text { Total Fuel Flow Rate } \\ \dot{m}_{\text {jet }} & \text { Mass flow rate from the Pre-chamber } \\ n & \text { Engine rotational speed } \\ \mathrm{P} & \text { Power } \\ \mathrm{S} & \text { Swirl angular momentum } \\ S_{L} & \text { Laminar Flame Speed }\end{array}$




$\begin{array}{ll}t & \text { Time } \\ \mathrm{T} & \text { Tumble angular momentum } \\ \mathrm{V} & \text { Volume }\end{array}$

\section{Greeks}

$\begin{array}{ll}\eta & \text { Efficiency } \\ \lambda & \text { Relative air-fuel ratio } \\ \Lambda_{T} & \text { Taylor length scale } \\ \rho & \text { Density } \\ \Sigma & \text { Turbulence-induced flame wrinkling } \\ \tau_{A I} & \text { Auto-ignition Time }\end{array}$

\section{References}

1. Nemeth, K.; Carvalho, J.M. Dieselgate and Consumer Law: Repercussions of the Volkswagen scandal in the European Union. J. Eur. Consum. Mark. Law 2017, 6, 35.

2. Sperling, D. Future Drive: Electric Vehicles and Sustainable Transportation; Island Press: Washington, DC, USA, 2013; ISBN 9781559633277.

3. Helms, H.; Pehnt, M.; Lambrecht, U.; Liebich, A. Electric vehicle and plug-in hybrid energy efficiency and life cycle emissions. In Proceedings of the 18th International Symposium Transport and Air Pollution, Empa, Dübendorf, Switzerland, 18-19 May 2010; p. 113.

4. Senecal, P.K.; Leach, F. Diversity in transportation: Why a mix of propulsion technologies is the wayforward for the future fleet. Results Eng. 2019, 4. [CrossRef]

5. Thiel, C.; Perujo, A.; Mercier, A. Cost and $\mathrm{CO}_{2}$ aspects of future vehicle options in Europe under new energy policy scenarios. Energy Policy 2010, 38, 7142-7151. [CrossRef]

6. Millo, F.; Rolando, R.; Fuso, R.; Zhao, J. Development of a new hybrid bus for urban public transportation. Appl. Energy 2015, 157, 583-594. [CrossRef]

7. Roberts, M. Benefits and Challenges of Variable Compression Ratio (VCR). SAE Tech. Pap. 2003. [CrossRef]

8. Francqueville, L.; Michel, J. On the Effects of EGR on Spark-Ignited Gasoline Combustion at High Load. SAE Int. J. Engines 2014, 7, 1808-1823. [CrossRef]

9. Bozza, F.; De Bellis, V.; Teodosio, L. Potentials of cooled EGR and water injection for knock resistance and fuel consumption improvements of gasoline engines. Appl. Energy 2016, 169, 112-125. [CrossRef]

10. Bozza, F.; De Bellis, V.; Teodosio, L.; Tufano, D.; Malfi, E. Techniques for $\mathrm{CO}_{2}$ emission reduction over a WLTC. A numerical comparison of increased compression ratio, cooled EGR and water injection. SAE Tech. Pap. 2018. [CrossRef]

11. Germane, G.; Wood, C.; Hess, C. Lean Combustion in Spark-Ignited Internal Combustion Engines-A Review. SAE Tech. Pap. 1983, 831694. [CrossRef]

12. Rapp, V.; Killingsworth, N.; Therkelsen, P.; Derek, D.-R. Lean Combustion, 2nd Edition. In Lean-Burn Internal Combustion Engines; Elsevier: Amsterdam, The Netherlands, 2016; pp. 111-146. [CrossRef]

13. Drake, M.; Fansler, T.; Lippert, A. Stratified-charge combustion: Modeling and imaging of a spray-guided direct-injection spark-ignition engine. Proc. Combust. Inst. 2005, 30, 2683-2691. [CrossRef]

14. Fajardo, C.; Sick, V. Flow field assessment in a fired spray-guided spark-ignition direct-injection engine based on UV particle image velocimetry with sub crank angle resolution. Proc. Combust. Inst. 2007, 31, 3023-3031. [CrossRef]

15. Stanglmaier, R.; Roberts, C. Homogeneous charge compression ignition (HCCI): Benefits, compromises, and future engine applications. SAE Trans. 1999, 2138-2145. [CrossRef]

16. Okita, R. Mazda SKYACTIV-G Engine with New Boosting Technology. In Proceedings of the Advanced Clean Cars Symposium: The Road Ahead, Air Resources Board, Sacramento, CA, USA, 27-28 September 2016.

17. Sens, M.; Binder, E.; Benz, A.; Kramer, L.; Schultalbers, M.; Blumenröde, K. Pre-chamber ignition as a Key Technology for Highly Efficient SI Engines-New Approaches and Operating Strategies. In Proceedings of the 39th International Vienna Motor Symposium, Vienna, Germany, 26-27 April 2018. 
18. Mueller, C.; Morcinkowski, B.; Habermann, K.; Uhlmann, T.; Schernus, C. Development of a pre-chamber for spark ignition engines in vehicle applications. In Proceedings of the 4th International Conference on Ignition Systems for Gasoline Engines, Berlin, Germany, 6-7 December 2018.

19. Bozza, F.; De Bellis, V.; Tufano, D.; Malfi, E.; Muller, C.; Habermann, K. A Quasi-Dimensional Model of Pre-Chamber Spark-Ignition Engines. SAE Tech. Pap. 2019. [CrossRef]

20. Bozza, F.; De Bellis, V.; Tufano, D.; Malfi, E.; Muller, C.; Habermann, K. 1D Numerical and Experimental Investigations of an Ultra lean Pre-Chamber Engine. SAE Int. J. Engines 2020. [CrossRef]

21. De Bellis, V.; Malfi, E.; Tufano, D.; Bozza, F. Efficient Thermal Electric Skipping Strategy applied to the Control of Series/Parallel Hybrid Powertrain. SAE Tech. Pap. 2020. [CrossRef]

22. Serrano, D.; Zaccardi, J.M.; Müller, C.; Libert, C.; Habermann, K. Ultra-Lean Pre-Chamber Gasoline Engine for Future Hybrid Powertrains. SAE Tech. Pap. 2020. [CrossRef]

23. Millo, F.; Bernardi, M.G.; Delneri, D. Computational Analysis of Internal and External EGR Strategies Combined with Miller Cycle Concept for a Two Stage Turbocharged Medium Speed Marine Diesel Engine. SAE Int. J. Engines 2011, 4, 1319-1330. [CrossRef]

24. Gottschalk, W.; Lezius, U.; Mathusall, L. Investigations on the Potential of a Variable Miller Cycle for SI Knock Control. SAE Tech. Pap. 2013. [CrossRef]

25. De Bellis, V.; Severi, E.; Fontanesi, S.; Bozza, F. Hierarchical 1D/3D approach for the development of a turbulent combustion model applied to a VVA turbocharged engine. Part II: Combustion model. Energy Procedia 2014, 45, 1027-1036. [CrossRef]

26. North, G.L.; Santavicca, D.A. The fractal nature of premixed turbulent flames. Combust. Sci. Technol. 1990, 72, 215-232. [CrossRef]

27. Blizard, N.; Keck, J. Experimental and Theoretical Investigation of Turbulent Burning Model for Internal Combustion Engines. SAE Tech. Pap. 1974, 740191. [CrossRef]

28. Hiraoka, K.; Nomura, K.; Yuuki, A.; Oda, Y.; Kameyama, T. Phenomenological 0-Dimensional Combustion Model for Spark-Ignition Natural Gas Engine Equipped with Pre-Chamber. SAE Tech. Pap. 2016. [CrossRef]

29. Bozza, F.; Teodosio, L.; De Bellis, V.; Fontanesi, S.; Lorio, A. A Refined OD Turbulence Model to Predict Tumble and Turbulence in SI Engines. SAE Int. J. Engines 2019, 12, 15-30. [CrossRef]

30. Bozza, F.; De Bellis, V.; Teodosio, L. A Tabulated-Chemistry Approach Applied to a Quasi-Dimensional Combustion Model for a Fast and Accurate Knock Prediction in Spark-Ignition Engines. SAE Tech. Pap. 2019. [CrossRef]

31. Bounaceur, R.; Herbinet, O.; Fournet, R.; Glaude, P.-A.; Battin-Leclerc, F.; da Cruz, A.P.; Yahyaoui, M.; Truffin, K.; Moreac, G. Modeling the Laminar Flame Speed of Natural Gas and Gasoline Surrogates. $S A E$ Tech. Pap. 2010. [CrossRef]

32. Lavoie, G.; Heywood, J.; Keck, J. Experimental and theoretical study of nitric oxide formation in internal combustion engines. Combust. Sci. Technol. 1970, 1, 313-326. [CrossRef]

33. Heywood, J. Internal Combustion Engine Fundamentals; Mcgraw-Hill: New York, NY, USA, 1988; Volume 930, ISBN 007028637X.

34. Kaplan, J.; Heywood, J. Modeling the spark ignition engine warm-up process to predict component temperatures and hydrocarbon emissions. SAE Tech. Pap. 1991, 361-376. [CrossRef]

35. Lavoie, G. Correlations of combustion data for SI Engine calculations-laminar flame speed, quench distance and global reaction rates. SAE Tech. Pap. 1978, 1015-1033. [CrossRef]

36. Bozza, F.; Tufano, D.; Malfi, E.; Teodosio, L.; Libert, C.; De Bellis, V. Performance and Emissions of an advanced Multi-cylinder SI engine operating in ultra-lean conditions. SAE Tech. Pap. 2019. [CrossRef]

37. Teodosio, L.; Pirrello, D.; Berni, F.; De Bellis, V.; Lanzafame, R.; D'Adamo, A. Impact of intake valve strategies on fuel consumption and knock tendency of a spark ignition engine. Appl. Energy 2018, 216, 91-104. [CrossRef]

38. Tufano, D.; De Bellis, V.; Malfi, E. Development of an on-line energy management strategy for hybrid electric vehicle. Energy Procedia 2018, 148, 106-113. [CrossRef]

39. Wipke, K.B.; Cuddy, M.R.; Burch, S.D. ADVISOR 2.1: A user-friendly advanced powertrain simulation using a combined backward/forward approach. IEEE Trans. Veh. Technol. 1999, 48, 1751-1761. [CrossRef]

40. Sciarretta, A.; Guzzella, L. Control of hybrid electric vehicles. IEEE Control. Syst. Mag. 2007, 27, 60-70. [CrossRef] 
41. Kim, N.; Cha, S.; Peng, H. Optimal control of hybrid electric vehicles based on Pontryagin's minimum principle. IEEE Trans. Control. Syst. Technol. 2011, 19, 1279-1287. [CrossRef]

42. Patil, R.M.; Filipi, Z.; Fathy, H.K. Comparison of Supervisory Control Strategies for Series Plug-In Hybrid Electric Vehicle Powertrains Through Dynamic Programming. IEEE Trans. Control. Syst. Technol 2014, 22, 502-509. [CrossRef]

43. Paganelli, G.; Delprat, S.; Guerra, T.M.; Rimaux, J.; Santin, J.J. Equivalent consumption minimization strategy for parallel hybrid powertrains. In Proceedings of the Vehicular Technology Conference IEEE 55th Vehicular Technology Conference VTC Spring, Birmingham, AL, USA, 7 August 2002; pp. 2076-2081. [CrossRef]

44. Commission Regulation EU, 2018/1832 of 5 November 2018, Official Journal of the European Union L 301/1. Available online: https://eur-lex.europa.eu/legal-content/EN/TXT/PDF/?uri=CELEX:32018R1832\&from=FR (accessed on 2 November 2019).

45. Valverde, V.; Michael, C.; Pierre, B.; Barouch, G.; Massimo, C.; Marcos, O.G.; Carsten, G.; Georgios, F.; Jrlica, P.; Giorgio, M.; et al. Joint Research Centre 2018 Light-Duty Vehicles Emissions Testing-Contribution to the EU Market Surveillance: Testing Protocols and Vehicle Emissions Performance EUR29897 EN; Publications Office of the European Union: Luxembourg, 2019; ISBN 978-92-76-12333-0. [CrossRef]

(C) 2020 by the authors. Licensee MDPI, Basel, Switzerland. This article is an open access article distributed under the terms and conditions of the Creative Commons Attribution (CC BY) license (http://creativecommons.org/licenses/by/4.0/). 